\title{
Digital diplomatics
}

The computer as a tool for diplomatist?

Edited by

ANTONELLA AMBROSIO, SÉBASTIEN BARRET, GEORG VOGELER

\section{ELEKTRONISCHER SONDERDRUCK}

\author{
Q \\ 2014 \\ BÖHLAU VERLAG KÖLN · WEIMAR · WIEN
}





\title{
Illuminierte Urkunden im digitalen Zeitalter Maßregeln und Chancen"
}

\author{
MARTIN ROLAND
}

Am Beginn dieses Beitrages wird der Untersuchungsgegenstand anhand einiger charakteristischer Beispiele vorgestellt (1) und ein Beispiel exemplarisch etwas ausführlicher vorgestellt (1a). Anschließend wird eine Definition versucht (2). Dann folgen praktische Beobachtungen und Vorschläge (Maßregeln), wie man illuminierte Urkunden konkret in Projekte einbinden könnte und welche Perspektiven und Chancen sich ergeben könnten.

\section{1) Beispiele illuminierter Urkunden}

Von ottonischer Zeit an gibt es Beispiele, bei denen gemalter „Buch“Schmuck Teil von Urkunden ist ${ }^{1}$. Nach derzeitigem Kenntnisstand stehen

"Die während des Vortrags in Neapel gezeigte englischsprachige PowerPointPräsentation, die naturgemäß über die hier abgedruckten Bildbeispiele hinausgeht, wird von der Ludwig-Maximilian-Universität in München unter der URL <http:// www.cei.lmu.de/digdipl11/slides-rep/Roland/index.pdf > zur Verfügung gestellt.

${ }^{1}$ Das Phänomen ist den Diplomatikern seit langem durchaus bewußt. Bereits in der Revue archéologique 1847/48 veröffentlichte L. DOUET-D’ARCQ einen Beitrag unter dem Titel „Chartes à vignettes. Représentation de Charles V“ (S. 749-756), der zwar nur französisches Material erwähnt, aber doch über die bekannten Beispiele aus der Regierungszeit von Karl V. (1364-80) hinausgreift. Einen profunden Überblick bietet A. GIRY in seinem erstmals 1871 erschienenen Manuel de diplomatique (vgl. S. 502-507 der Auflage von 1925 [diese digitalisiert auf gallica.bnf.fr]). W. WATTENBACH, Schriftwesen im Mittelalter ( $\left.{ }^{1} 1871\right)$, weitet den Blick (erstmals?) über nationale Grenzen (vgl. die ganz kurzen Bemerkungen S. 221), die dritte Auflage (1896) enthält auf S. 383f. bereits eine ansehnliche Beispielfolge. Eine bemerkenswerte Initiative von Seiten der Archive stellt die Einrichtung und Publikation des Musée des Archives nationales. Documents originaux de l'histoire de France exposés dans l'Hôtel Soubise, introduction par A. MAURY (1872), dar, dem 1878 der Band Musée des Archives départementales folgte, der statt Nachstichen bereits photographische Abbildungen enthielt. Zur rezenteren Forschung siehe S. 261. An den Archives nationales wurde ein eigenes Selekt mit "chartes à vignettes“ (illuminierten Urkunden) eingerichtet; vgl. 
zwei Urkunden aus der Kanzlei der ottonischen Kaiser am Beginn der Entwicklung: das sogenannte Ottonianum für die römische Kirche von $962^{2}$ und die berühmte Dotalurkunde für Theophanu, der byzantinischen Gemahlin Kaiser Ottos II., von 972, deren Oberfläche wie ein byzantinischer Seidenstoff gestaltet ist. Die Urkunde wurde in Rom anläßlich der Hochzeitsfeierlichkeiten ausgestellt und war offenbar ein zentrales Element der Selbstdarstellung des abendländischen Kaisertums gegenüber Byzanz ${ }^{3}$.

Aus dem 11. Jahrhundert habe ich für diesen Überblick eine weitere „Hochzeitsurkunde“ ausgewählt, die exemplarisch ausführlicher behandelt werden soll, um an einem ganz konkreten Beispiel das interdisziplinäre Potential aufzuzeigen, das das Studium illuminierter Urkunden birgt.

\section{1a) Magister Mel und Alfarana}

Die Theophanu-Urkunde enthält erstmals figürliche Elemente, deren Verbindung zum Rechtsinhalt freilich als eher indirekt zu klassifizieren ist. Bei der nun zu besprechenden Urkunde ${ }^{4}$ sind die Darstellung - ein Hochzeitspaar - und das

Images du pouvoir royal. Les chartes décorées des Archives nationales XIII ${ }^{\mathrm{e}}-\mathrm{XV}{ }^{\mathrm{e}}$ siècle, hg. von G. BRUNEL (2005) S. 36.

2962 Februar 13, Rom; Rom, Archivio Segreto Vaticano, Arm. 1, Caps. III, nr. 1, = MGH D O.I. 235). - Die Theophanu-Urkunde wird im Folgenden als Teil eines performativen Geschehens interpretiert; die Hinterlegung des Ottonianums in der Peterskirche wird ebenfalls keine auf das Juridische beschränkte Handlung, sondern eine theatralische Inszenierung gewesen sein. Der Zusammenhang zwischen besonders gestalteter äußerer Form der Urkunde und der Gestaltung des „Events“ ist sicherlich kein Zufall.

3972 April 14, Rom; Wolfenbüttel, Niedersächsisches Staatsarchiv, 6 Urk. 11, = MGH D O.II. 21). Der Sachinhalt ist bedeutend aber durchaus nicht außergewöhnlich. Entsprechende Dotalurkunden gab es schon für Adelheid, die spätere Mutter Ottos II., die deren erster Mann Lothar von Italien zusammen mit seinem Vater Hugo 937 Dez. 12, Colombier, für sie ausgestellt hatte. - Die Form - Goldschrift und Purpurgrund - weist auf Staatspolitisches. Wurde im Ottonianum (siehe Anm. 2) das Verhältnis zur römischen Kirche bestimmt, thematisiert die Form der „Hochzeitsurkunde“ das Verhältnis des westlichen Kaisertums zu Byzanz, indem selbstbewußt byzantinische Anregungen rezipiert und umgestaltet werden (Mustergrund mit Tierkampfmedaillons). Zum Verhältnis der beiden Urkunden zu byzantinischen Auslandschreiben vgl. die umfangreichen Angaben in: M. Roland/A. ZaJiC, Les chartes médiévales enluminées dans les pays de l'Europe centrale, in: BECh 169 (2011) [2013 erschienen] S. 151-253, bes. S. 153-158; vgl. Anm. 57.

${ }^{4}$ Bari, Archivio del Capitolo Metropolitano, Perg 14: G. NitTo De Rossi/F. NitTi DI Vito, Le pergamene del Duomo di Bari (Codice diplomatico Barese 1, 1897) S. 24 (Nr. 14); in der online verfügbaren Datenbank Pergamene di Puglia online <http:// www.sapuglia.it/index.php?option=com_content $\&$ view $=$ article $\&$ id $=46 \&$ Itemid $=66>$ ist unter <http://www.sapuglia.it/Schedatura/02_mostra_dati_front.php?id_ perg=1153\&pag=1 > das Digitalisat des Eintrags im genannten Urkundenbuch und das 
Rechtsgeschäft offenkundig und ganz unmittelbar aufeinander bezogen (Abb. IV, Farbtafelteil): Magister Mel filius Natalis de civitate Vari (Bari), Vorort der byzantinischen Verwaltung in Süditalien ${ }^{5}$, heiratete im Dezember des Jahres $1028^{6}$ Alfarana filia Bisantii de predicta civitate ${ }^{7}$, und übergab ihr die bis heute

Digitalisat der Urkunde selbst verlinkt (Zugriff jeweils 5.9.2013). Grundlegend M. Cannataro, Un insolito documento privato Barese del secolo XI, in: Annali della Facoltà di lettere e filosofia 19-20 (1976/1977) S. 203-221; H. BeLTing, Studien zur beneventanischen Malerei (1968) S. 186f.; Alle sorgenti del Romanico, hg. von P. BeLLi D’EliA, Ausstellung Bari 1975, S. 112 (Kat.-Nr. 128: W. F. Volbach) und Tafel II. Neben der älteren Literatur, die im Katalog von 1975 erwähnt ist, vgl. Medioevo. La chiesa e il palazzo. Atti del Convegno internazionale di studi, Parma, 20-24 settembre 2005, hg. von A. C. Quintavalle (2007) S. 242, und A. Amati Canta/L. Sinisi, Il dono del mattino, in: 1087 i costumi della traslazione - donne, gioielli e promesse nuziali, hg. von L. SpezZacatene (2011) S. 57-68. Zu diesem Ausstellungsprojekt siehe auch Anm. 29.

${ }^{5} \mathrm{Zu}$ den historischen Umständen vergleiche V. von FalkenHausen, Untersuchungen über die byzantinische Herrschaft in Süditalien vom 9. bis ins 11. Jahrhundert (1967).

${ }^{6}$ Die Datierung des Stückes ist problematisch: Es werden die Herrscherjahre Konstantins VIII. angegeben (nämlich das 69. Jahr), die Indiktion (nämlich 11) und der Monat (nämlich Dezember). Offenbar konsequent wurden bei allen Bareser Urkunden die Herrscherjahre ab 960 gezählt, dem Jahr in dem Basileios (der Bruder des genannten Konstantin) am 22. April Mitkaiser wurde. Oft wird der jüngere Bruder ebenfalls erwähnt, was jedoch (spätestens ab der Jahrtausendwende) nichts an der (gemeinsamen) Zählung ändert. Die Zählung blieb auch bestehen, nachdem Konstantin alleine herrschte, obwohl er erst zwei Jahre nach seinem älteren Bruder Mitkaiser geworden war (30. März 962). Bemerkenswert ist nun, daß die Relation zwischen Kaiserjahren und Indiktion, die dem griechischen Gebrauch folgend am 1. September wechseln sollte, egal in welchem Monat die Urkunde ausgestellt wurde, immer konstant bleibt. Der Wechsel scheint sich, wenn ich das Material aus der Regierungszeit der beiden Brüder richtig deute, jeweils am 1. Jänner vollzogen zu haben. Daraus ergibt sich für unsere illuminierte Urkunde, wie schon die Editoren des Jahres 1897 richtig erkannt hatten, eine Entstehung im Dezember 1028 und nicht (wie von Maria Cannataro [siehe Anm. 4] S. 203 behauptet) im Dezember 1027.

7 Die Bezeichnung ,Magister' für den Bräutigam deutet wohl auf eine angesehene handwerkliche Tätigkeit hin. Er tritt in zwei weiteren Urkunden (1030 bzw. 1031) auf und wird dort als Mel ferrarius bzw. als Mel magister ferrarius bezeichnet (CDB 1, wie Anm. 4, Nr. 16f.); der Name seines Vaters (Natalis) sichert die Identität des Mannes, der mit einem gleichnamigen Verwandten ein Grundstücksgeschäft abwickelt. So werden auch die verschiedenen Metalle verständlich, die bei der an sich formelhaften Aufzählung der Besitztümer vorkommen (de auro vel argento, here vel ferro, stagno vel plumbo). Alfarana entstammt einer angesehenen, mit der bzyantinischen Verwaltung eng verbundenen Familie; detailliert zu ihrer sozialen Stellung Cannataro, Documento (wie Anm. 4) S. 214-218. Daß auch die Darstellung selbst einen sozialen Unterschied deutlich macht (so Cannataro S. 218), kann ich bei bestem Willen nicht erkennen. 
erhaltene Urkunde und ein Viertel seines Besitzes als morgincaph ${ }^{8}$. Die Zeremonie wurde secundum ritus gentis nostre La(n)gobardorum vollzogen und in dem über weite Strecken formelhaften Vertrag festgehalten'. Die Schriftformen der Urkunde des Notars Pandus ${ }^{10}$ sind stark buchschriftlich geprägt ${ }^{11}$,

${ }^{8} \mathrm{Zu}$ den auf langobardischem Recht beruhenden eherechtlichen Bedingungen in Bari vgl. L. TRIA, La disciplina giuridica del matrimonio secundo le consuetudini di Terra di Bari, in: Iapiga 7 (1936) S. 392-419, bes. S. 409, 413-415, und 8 (1938) S. 19 62, bes. S. 24-32; A. Аmati Canta, Meffium, morgincap, mundium. Consuetudini matrimoniali longobarde nella Bari medievale (2006; vgl. auch „Morgengabe“ im Deutschen Rechtswörterbuch 2004ff, <http://drw-www.adw.uni-heidelberg.de/drwcgi/zeige? $\mathrm{db}=\mathrm{dr}$ \& \&index $=$ lemmata\&term $=$ Morgengabe\&darstellung $=\% \mathrm{DC}>(\mathrm{mit}$ vielen Verweisen).

${ }^{9}$ Das hier besprochene Stück scheint der älteste Beleg für ein offensichtlich für Bari und einige Orte in dessen Umgebung (Conversano 1181, XII; Acquaviva 1373, V 11) charakteristisches Diktat zu sein, das - mit gewissen Varianten - bis ins 14. Jahrhundert verwendet wurde; vergleiche die Belege in der Datenbank Pergamene di Puglia online (wie Anm. 4), Stichwort „morgincap“. Die für die Urkunde von 1028 so typische Aufzählung von Besitztümern (vgl. TRIA, disciplina [wie Anm. 8] 8 [1938] S. 26f.) findet sich (mit deutlichen Abweichungen) sonst nur in einer Urkunde von September 1060. Eine einzige der Urkunden (1202 XII 3, Bari, Protonotar Lupo) weist (nicht historisierten) Buchschmuck auf, nämlich Palmettenfleuronnée und zoomorphe Elemente (siehe Anm. 72); zu Palmettenfleuronnée auf Urkunden vgl. Abb. IX und den Abschnitt zu den Urkunden Kaiser Friedrichs II. auf S. 263 f. zu weiteren Notaren, die ihre Dokumente mit Buchschmuck ausstatteten siehe Anm. 73.

${ }_{10}$ Zum Notar Cannataro, Documento (wie Anm. 4) S. 207f. und F. Magistrale, Notariato e documentazione in Terra di Bari. Ricerche su forme, rogatari, credibilità dei documenti latini nei secoli IX-XI (1984). Pandus ist auch aus anderen Urkunden bekannt (bis Oktober 1065), in keiner derselben verwendet er jedoch eine vergleichbar hoch stilisierte Schrift (CANNATARO Anm. 9). Sein unauffälliges Signet, wie üblich als Abschluß der letzten Textzeile, ist aus mehreren zu einem Konglomerat vereinten Spirallinien gebildet und (nur in dieser Urkunde) rot-violett und grün koloriert. Über den eigenhändigen Anteil des Notars, über die ihm zu Gebote stehenden Schriftgrade und die Frage, ob er vielleicht auch der Zeichner war (wie CANNATARO S. 207 glaubt), ist sicher noch nicht das letzte Wort gesprochen.

${ }^{11}$ Ausführlich zur Schrift und deren Verhältnis zu Buchschriften Cannataro, Documento (wie Anm. 4) S. 208-213, die S. 209 unter anderem eine Urkunde von April 1024 nennt (CDB 1, wie Anm. 4, Nr. 12). Auf paläographische Verbindungen zwischen der Exultet-Rolle „Bari 1“ (siehe Exultet. Rotoli liturgici del medioevo meridionale, hg. von G. Cavallo [1994] S. 129-141, bes. S. 134) und zwei Bareser Urkunden weist Francesco Magistrale in seiner Beschreibung der genannten Rolle hin; er nennt die Urkunde von April 1024 und ein Stück von November 1028 (Bari, Archivio di S. Nicola, B7; CDB 4, Nr. 19 [hier 1029]). Beide weisen tatsächlich buchschriftlichen Charakter auf und ragen damit aus der Gesamtüberlieferung hervor. Unsere Urkunde könnte hier durchaus auch genannt werden, zeichnet sich jedoch zusätzlich durch die weiten Zeilenabstände aus. 
als erster der fünf Zeugen unterfertigt Joannes (Proto-)Spatarios und Turmarca die Urkunde ${ }^{12}$.

Während all dies dem zeitgenössischen Urkundenwesen durchaus entspricht, stellt die kolorierte Federzeichnung ${ }^{13}$ in der Mitte des Urkundentextes eine, wie schon erwähnt, zumindest aus heutiger Sicht erstmalige, jedenfalls jedoch herausragende Leistung dar. Das Bildfeld wurde bereits vor der Mundierung festgelegt, denn die Schrift nimmt auf den unregelmäßigen Umriß der Darstellung Rücksicht ${ }^{14}$. Von einer Arkade mit zwei übergroßen Vögeln umgeben tritt der Betrachterin / dem Betrachter das Brautpaar entgegen $^{15}$, das gemeinsam einen länglichen Gegenstand hält, der auf Grund der Falten textil wirkt ${ }^{16}$.

${ }_{12}$ Dieser hohe byzantinische Repräsentant und ein weiterer Zeuge verfügen über geübte griechische Schriftformen, die drei weiteren Zeugen verwenden das lateinische Alphabet. Ob es derselbe Amtsträger war, der bei der Festlegung der Grenzen des Territoriums von Troia im Juni 1019 anwesend war (vgl. von Falkenhausen, Byzantinische Herrschaft [wie Anm. 5] S. 177-179 [Nr. 41]), müßte noch geklärt werden.

${ }^{13}$ Die Farben treten auch bei der Ausschmückung von Elementen der Schrift auf und machen daher einen einheitlichen Entstehungsprozeß (nicht zwingend aber Handgleichheit) wahrscheinlich. Bemerkenswert ist, daß auch das Notariatssignet des Pandus koloriert wurde. Die grüne Farbe hat das Pergament stark angegriffen. Besonders deutlich wird dies bei den beiden Säulen der Arkade und dem Bodenstreifen, der sich über die gesamte Breite der Urkunde erstreckt.

${ }^{14}$ Dies ist übrigens ein Phänomen, das Jahrhunderte später auch bei den Wappenbriefen der Reichskanzlei, deren Bildfeld ebenfalls inmitten der Schrift plaziert ist, beobachtet werden kann.

${ }^{15}$ Ikonographisch gibt es aus so früher Zeit kaum Vergleichbares: Kaiserpaare wurden zwar häufiger dargestellt, sind jedoch nur sehr bedingt als Parallele zu betrachten. Hinzuweisen ist auf das königliches Paar im karolingischen Stuttgarter Psalter (Stuttgart, Württembergische Landesbibliothek, Cod. bibl. $2^{\circ}$ 23, fol. 57v [zu Ps. 45]; siehe M. Müller, Die Kleidung nach Quellen des frühen Mittelalters [2003] S. 104 und Abb. 1), auf das Benedictionale des Erzbischofs Landolf von Benevent (Rom, Biblioteca Casanatense, Cas. 724/2, Sektion 5c [Hochzeit zu Kana; Exultet (wie Anm. 11)] S. 95) und auf ein (nach $1087 \mathrm{zu}$ datierendes) Fresko in der Grotta dei santi in Calvi, das als Stifter ein Ehepaar zeigt (Belting, Studien [wie Anm. 4] S. 105-111 und Abb. 120). Während das karolingische Beispiel den Bräutigam in kurzer Tunika zeigt, kleiden ihn die beiden späteren Darstellungen - so wie unsere Urkunde - in fußlange Tuniken.

${ }^{16}$ Das Urkundenformular erwähnt ausdrücklich die Übergabe der Urkunde, entsprechend wurde die Darstellung bisher auch durchgehend (und wohl auch zu Recht) interpretiert. Freilich weisen weder die kompositorischen Mittel klar auf eine Übergabe hin, noch ist das Objekt als Schriftrolle klar zu erkennen. Besonders das zweite Argument wiegt schwer, weil in den Exultet-Rollen Bildformeln für Schriftrollen ganz geläufig sind. Sollte es sich jedoch tatsächlich um ein Textil handeln, wäre auf (bis ins 20. Jahrhundert belegte) Bräuche hinzuweisen, die das blutige Leintuch der Hochzeitsnacht zur Schau stellten. 
Bemerkenswert ist die Darstellung der Kleidungsstücke, vor allem weil diese fest lokalisiert und datiert und zudem einer ganz spezifischen Handlung zuzuordnen ist. Dies ist umso spannender, weil in schriftlichen Quellen der Kleidung oft eine ethnische Gruppen unterscheidende Funktion zugeschrieben wird ${ }^{17}$. So thematisiert etwa die um 1100 entstandene Gesta Roberti Wiscardi des Wilhelm von Apulien (Guilelmus Apuliensis) die Wirkung von fremdartiger Kleidung erstaunlich prominent ${ }^{18}$ : Kann man anhand realienkundlicher Vergleiche die Kleidung unseres Brautpaares einer der damals in Bari lebenden Gruppen zuordnen oder entlarvt die Darstellung entsprechende Texte als ideologische Konstrukte der Verfasser?

Mechthild Müller gibt einen Überblick über die Entwicklung der Kleidung im Westen, Maria Parani in Byzanz ${ }^{19}$. Keines der bei unserem Brautpaar zu sehenden Stücke ist nur mit byzantinischen Quellen zu belegen: weder die mit reichen Borten besetzten Tuniken an sich (Abb. IVc) ${ }^{20}$, noch

${ }^{17}$ MÜLlER, Kleidung (wie Anm. 15) S. 65, 170-172 zitiert verschiedene Belege, die herrscherliche Kleidung ethnischen Gruppen (Franken, Griechen) zuordnen, wobei Zweiteres von den Autoren in der Regel negativ konnotiert wird. Grundsätzlich zum Verhältnis von ethnischer Zuordnung und Kleidung PH. von RummeL, Gotisch, barbarisch oder römisch? Methodologische Überlegungen zur ethnischen Interpretation von Kleidung, in: Archaeolgy of Identity - Archäologie der Identität, hg. von W. Pohl/M. Mehofer (Forschungen zur Geschichte des Mittelalters 17, 2010) S. 51-77, bes. S. 64-74.

${ }^{18}$ Edition des Textes in: Guillaume de Pouille, La Geste de Robert Guiscard, hg. von M. Mathieu (1961) Buch 1, Verse 13-16. Davor wird die Fremdheit der Sprache der neu ins Land kommenden Normannen thematisiert, dann, als erste Szene, begegnen die Normannen als Pilger auf dem Monte Gargano dem (langobardischen) Rebellen Mel, der sich gegen die byzantinische Herrschaft (erfolglos) erhoben hatte. Mel wäre, so der Autor, more virum Graeco vestitum gewesen.

${ }^{19}$ Müller, Kleidung (wie Anm. 15); M. G. PArani, Reconstructing the Reality of Images. Byzantine Material Culture and Religious Iconography $\left(11^{\text {th }}-15^{\text {th }}\right.$ Centuries) (2003) S. 11-100, bes. S. 54f. und 72f. (zur mittelbyzantinischen Tunika), S. 67f. (zu männlichen Kopfbedeckungen; mit Abb. 77) und S. 79f. (zu Ohrringen).

${ }^{20}$ Hier ist auf die ottonische Buchmalerei im allgemeinen (und deren Aufarbeitung durch Müller, Kleidung [wie Anm. 15]) und im speziellen auf die westlichen Beispiele in den folgenden Anmerkungen und auf die in Benevent (also außerhalb des byzantinischen Einflußgebietes) in den 980er Jahren entstandene Exultet-Rolle im Vatikan (Biblioteca Apostolica Vaticana, Vat. lat. 9820) zu verweisen, wo ein Fürst und die mit ihm dargestellten Personen derartig ausgestattete (freilich nur knielange) Tuniken tragen (Exultet, wie Anm. 11, S. 111 und Müller S. 172f.). Müller leitet die lange Herrschertunika aus Byzanz ab, betont aber wohl zu stark die Dominanz der kurzen Tunika, die sie für den Regelfall hält, während lange nur vom Herrscher getragen würden (z. B. S. 181). Unsere Darstellung ist ein klares Gegenbeispiel. - Ein entsprechendes Kleidungsstück, die sogenannte Tunika Kaiser Heinrichs II. (Domschatz Bamberg; vor 1127; Abb. IVc , Müller S. 271 und K. KANIA, Kleidung im Mittelalter. 
Details wie die Oberarmborte ${ }^{21}$ am rechten Arm des Bräutigams, die untere Borte seiner Tunika mit dem vertikalen Teil in der Mitte ${ }^{22}$ noch die Brautkrone $^{23}$ und die Ohrringe ${ }^{24}$ der Alfarana. Ganz im Gegenteil, wollten wir der

Materialien - Konstruktion - Nähtechnik. Ein Handbuch [2010] S. 276), ist sogar erhalten geblieben.

${ }^{21}$ Parani, Reconstructing (wie Anm. 19) S. 54 (mit diversen Bildbeispielen) kennzeichnet Oberarmborten als mittelbyzantinische Entwicklung. Entsprechende Darstellungen gibt es freilich auch (und wohl sogar früher) im Westen; z. B. die Darstellung der Roma im sogenannten Evangeliar Ottos III. (München, Bayerische Staatsbibliothek, Clm 4453, fol. 23v).

${ }^{22}$ Dabei könnte es sich um die etwas unklare Wiedergabe einer in Byzanz belegten Besonderheit des 11. Jahrhunderts handeln, nämlich eine kürzere, vorne zu öffnende Tunika über einer geschlossenen unteren zu tragen; vgl. PARANI, Reconstructing (wie Anm. 19) S. 54f. und Tafel 26; vergleiche auch entsprechende Figuren im Madrider Skylitzes (Madrid, Nationalbibliothek, Cod. Vitr. 26-2, z. B. foll. 23r, 164r [farblich der Urkunde vollkommen entsprechend], 222r), bei denen (wie bei der Urkunde) nicht mehr deutlich ist, ob es sich tatsächlich um zwei Kleidungsschichten oder bloß um eine besondere Führung der unteren Borte handelt. - Der reich illustrierte Codex der Synopsis Historion des Ioannes Skylitzes (verfaßt um 1070/80, über Ereignisse von 811-1057 berichtend) ist nach einem byzantinischen Vorbild des 4. Viertels des 11. Jahrhunderts im 3. Viertel des 12. Jahrhunderts in San Salvatore in Messina entstanden. Ausführlich behandelt in: M. Roland, Zeitgeschichte ins Bild gesetzt. Ein Versuch zur Materialsammlung bis ca. 1450 (noch unpubliziert).

${ }^{23}$ Ph. Ditchfield, La culture matérielle médiévale. L'Italie méridionale byzantine et normande (2007) S. 455f. und 482, verwendet unsere Illustration, um die Brautmode $\mathrm{zu}$ beschreiben, und beschreibt die Kleidung der Braut (nicht aber jene des Mannes) als charakteristisch für die Zeremonie. Dies mag für die (Braut-)Krone mit Kreuz zutreffen (eine Krone ohne Kreuz in der Anm. 15 erwähnten Darstellung der Hochzeit von Kana), für die Kleidung selbst wäre dies jedoch erst mit entsprechenden Abweichungen zur üblichen weiblichen Kleidung zu belegen. Der Kronreifen im Kathedralschatz von Bari (13. Jh.?), den Ditchfield S. 482 nennt, hat keine erkennbare Beziehung zu Hochzeitszeremonien.

Die Krönung als Teil der Hochzeitszeremonien ist sowohl in der griechischen als auch in der lateinischen Kirche weit verbreitet. Weder ein von Amati CANTA/Sinisi, Dono del mattino (wie Anm. 4) S. 61 genanntes byzantinisches Beispiel (10. Jh.), bei dem die Verwendung ganz ungesichert ist, noch zwei westliche Brautkronen aus dem 15. Jahrhundert (der Hedwig von Polen [1475; Passau, Oberhausmuseum] und der Mechthild von Hessen [1489, Soest, Burghofmuseum]) weisen irgendwelche formalen Parallelen auf.

${ }^{24}$ Ohrringe aus dem byzantinischen Reich sind reichlich überliefert, sowohl original als auch in Bildquellen. Man vergleiche die vorbildliche Studie von A. BosseLMANN-RUICKBIE, Byzantinischer Schmuck des 9. bis frühen 13. Jahrhunderts. Untersuchungen zum metallenen dekorativen Körperschmuck der mittelbyzantinischen Zeit anhand datierter Funde (2011), zum Ohrschmuck S. 98-111, 125 (Fundumstände weibliche Gräber), 128-131 (zu den Darstellungen). Zu den von Alfarana getragenen halbmondartig geformten Ohrringen vgl. BosselmanN-Ruickвie S. 104-107, Abb. 96128, S. 342-366 (vergleichbare Stücke im Objektkatalog, bes. S. 253, Kat. 68 [für 
Chronologie Paranis folgen, dürfte es die weiten (von Müller als tütenförmig beschriebenen) Ärmel von Alfaranas Kleid erst ab der Mitte des 11. Jahrhunderts geben ${ }^{25}$. Tragfähige Hinweise auf eine Differenzierung von Kleidung nach (ethnischer) Gruppenzugehörigkeit kann die Realienkunde (zumindest in diesem Fall) nicht erbringen.

Abschließend ist noch nach stilistischen Vergleichen zu fragen ${ }^{26}$. Die Ableitung der Technik (kolorierte Federzeichnung) und die stilistischen Grundlagen sind mit den frühen liturgischen Rollen aus Benevent unbestritten ${ }^{27}$. Als sowohl örtlich wie chronologisch nächstliegender Vergleich bietet sich die Exultet-Rolle „Bari 1“ an $^{28}$. Wenn man etwa die einfachen, vom Gürtel

Kreta, jedenfalls vor 961], zu den Fundumständen vgl. 41-44). Bei den bildlichen Quellen ist nicht nur die schiere Menge sondern auch Objekte bemerkenswert, die das einseitig offene Tragen der halbmondartigen Ohrringe zeigen (S. 342, D1; S. 345f., D14, S. 348, D22). Die Überlieferungslage ist freilich auch in Süditalien ab der Spätantike breit; vgl. unmittelbar entsprechende Funde bei Ditchfield, Culture matérielle (wie Anm. 23) S. 496 und Abb. 69 und ausführlich und sehr ausgewogen C. D’ANGELA, Le oreficerie bizantine del museo nazionale di Taranto, in: Vetera Christianorum 21 (1984) S. 181-196, bes. S. 182 (Nr. 1), S. 183 (Nr. 5) und umfassend zum Typus S. 187192. D’Angela bestätigt den unmittelbaren Einfluß aus Byzanz, will aber eine lokale Produktion trotz des hohen Qualitätsniveaus wegen der ganz einheitlichen Machart der diversen Stücke nicht ausschließen. Sehr ähnliche Ohrringe trägt auch die Braut der Hochzeit von Kana in der Anm. 15 erwähnten Darstellung. Amati CanTA/SinisI, Dono del mattino (wie Anm. 4) 61 können belegen, daß Ohrringe in Bari auch als Hochzeitsgeschenke dienten.

${ }^{25}$ Parani, Reconstructing (wie Anm. 19) S. 73 (mit Tafel 81-83). Da jedoch in der zeitgleichen Exultet-Rolle „Bari 1“ (siehe Anm. 28) eindeutig erkennbare weite Ärmel vorkommen (Sektion 3a: Darstellung der Tellus; Exultet [wie Anm. 11] S. 137), ist die Chronologie nicht aufrecht zu erhalten (weitere frühe westliche Beispiele bei Müller, Kleidung [wie Anm. 15] S. 185f., 188-190 und z. B. Abb. 57). - Dieser Befund wird auch nicht beeinflußt von der unklaren Darstellung der Kleidung der Alfarana: Statt weiter Ärmel könnte durchaus auch ein kurzer Mantel über einer Tunika dargestellt sein (vgl. dazu Parani S. 84 und als Beispiel Abb. 80).

${ }^{26}$ Der grundlegende Aufsatz von Maria Cannataro widmet dieser Frage kaum Aufmerksamkeit. Die Autorin verweist auf eine augenscheinlich irrige Zuschreibung des Paläographen Armando Petrucci an den Zeichner der Exultet-Rolle „Bari 2“: Cannataro, Documento (wie Anm. 4) S. 205f.; Zur Exultet-Rolle (Bari, Archivio del Capitolo Metropolitano, Exultet 2) siehe Exultet (wie Anm. 11) S. 201-210 (F. MAGISTRALE; vollständig abgebildet).

${ }^{27}$ Diese wurden für bzw. in unmittelbarer Nachfolge des (Erz-)Bischofs Landolf von Benevent (Ernennung zum Erzbischof im Jahr 969) hergestellt; vgl. Exultet (wie Anm. 11) S. 75-118 sowie z. B. Alle sorgenti del Romanico (wie Anm. 4) S. 112 (Kat.Nr. 128: F. Volbach).

${ }^{28}$ Bari, Archivio del Capitolo Metropolitano, Exultet 1; vgl. Exultet (wie Anm. 11) S. 129-141 (F. Magistrale; vollständig abgebildet). Die Darstellung zweier byzantinischer Kaiser als weltliche Autorität (Sektion 7b) weist auf Basileios II. und seinen 
ausgehenden Faltenlinien und die graphischen Gesichtsformeln in der Darstellung der liturgischen Szene (Sektion 3b) betrachtet, dann spricht einiges für die Nähe (sicher jedoch nicht für die Handgleichheit) der Ausführung.

Zusammenfassend ist - wie bei der Theophanu-Urkunde - auch bei dem Stück aus Bari, eine kulturelle (Byzanz, Westen) und (hier zusätzlich) eine soziale Gemengelage (Mel als Aufsteiger) zu konstatieren. Stilistisch und paläographisch kann das Stück von 1028 gut als Fixpunkt in die jeweiligen Entwicklungen eingebunden werden. Unsere Analyse hat die starke Interdisziplinarität gezeigt, die bei der Beschäftigung mit illuminierten Urkunden gefordert ist. Ein Ausstellungsprojekt des Jahres 2011 hat zudem belegt, daß auch die zeitgenössische Kunstszene von einem derartigen Objekte profitieren kann (Abb. IVb) ${ }^{29}$.

Aus dem 12. Jahrhundert können eine Urkunde des schottischen Königs Malcolm IV. von $1159 / 60^{30}$, ein französischer Chirograph von $1177^{31}$ und eine Bruderschaftsurkunde von 1195 aus Saint-Martin de Canigou in den Pyrenäen $^{32}$ angefügt werden. Als Beispiele des 13. Jahrhunderts erwähne

jüngeren Bruder Konstantin VIII., die bis zum Tod des älteren 1025 nominell gemeinsam regierten. Zwar sind solche Darstellungen mitunter aus Vorlagen übernommen worden (wie Magistrale in Bezugnahme auf einen [in vielerlei Hinsicht nicht mehr grundlegenden] Aufsatz von Gerhard B. Ladner aus dem Jahre 1952 argumentiert), er selbst nennt jedoch gut nachvollziehbare paläographische Vergleiche, die in die (späteren) 1020er Jahre weisen. Eine Datierung um 1025 erscheint daher, gegen die übliche Spätdatierung auch wegen des Stilvergleichs mit unserer Urkunde am wahrscheinlichsten. Eine Spätdatierungen z. B. in: Medioevo, hg. von Quintavalle (wie Anm. 4) S. 242 (als Vergleich eine Exultet-Rolle im Museo dell'Opera del Duomo in Pisa [Exultet 2]; siehe Exultet [wie Anm. 11] S. 151-174 [A. R. Calderoni MaseTi; vollständig abgebildet], wo die Rolle unter Vorbehalt nach Capua lokalisiert und um 1059-1071 datiert wird).

${ }^{29}$ Die Kostüme wurden im Rahmen eines bemerkenswerten künstlerischen Ausstellungsprojekts nachgeschneidert (Abb. IVb); vgl. den Ausstellungskatalog (siehe Anm. 4) und <http://www.1087.eu> (Link derzeit nicht verfügbar).

30 E. Danbury, Décoration et enluminure des chartes royales anglaises au Moyen Âge, in BECh 169 (2011) [2013 erschienen] S. 79-107.

${ }^{31}$ Chirograph des Mathieu III., Grafen von Beaumont und des Abtes Geoffrey von Saint-Martin in Pontoise, bei dem eine Federzeichnung des Gekreuzigten als Beglaubigungszeichen diente (1177; Paris, Archives nationales, J 168, Nr. 2: B. M. BedosREZAK, Cutting Edge. The Economy of Mediality in Twelfth-Century Chirographic Writing, in: Das Mittelalter. Perspektiven mediävistischer Forschung 15 (2010) S. 134161 (mit sicher viel zu weitreichender Interpretation); P. BuREAU, Couper le corps du Christ en deux. Un chirographe imagé du XII ${ }^{\mathrm{e}}$ siècle, in: Revue française d'héraldique et de sigillographie 71-72 (2001/02) S. 153f.

32 Roland/Zajic, Chartes médiévales enluminées (wie Anm. 3) Anm. 33. 
ich die Urkunde der Lupusbrüder in Köln von $1246^{33}$ und eine englische Königsurkunde von 1291, deren Schmuck zwar keine menschlichen Figuren, aber mit den jagdbaren Tieren im unteren Bereich doch einen eindeutigen Bezug zum Inhalt der Urkunde zeigt ${ }^{34}$.

So wie in England, wo seit dem späteren 13. Jahrhundert eine kontinuierliche Produktion illuminierter Urkunden zu beobachten ist ${ }^{35}$, dominiert auch in Frankreich der König und sein Umfeld. Kunsthistorisch bemerkenswert sind Beispiele aus der Regierungszeit von Karl V. (1364-80) ${ }^{36}$. Dieses Portrait aus einer Urkunde von 1367 (Abb. Va) ${ }^{37}$ steht dem berühmten Tafelbild (Abb. Vb) um nichts nach, es ist vielmehr fest datiert und der Dargestellte ist unzweifelhaft, zwei Eigenschaften, die die Urkunde dem Tafelbild sogar voraus hat ${ }^{38}$.

${ }^{33}$ Ausführlich analysiert in Roland/ZajIC, Chartes médiévales enluminées (wie Anm. 3) S. 9f.

${ }^{34}$ A. H. ZaJic/M. Roland, Eine spätmittelalterliche Urkundenfälschung aus dem Augustiner-Chorherrenstift Dürnstein in Niederösterreich, zugleich ein Beitrag zu illuminierten Urkunden des Mittelalters in: AfD 51 (2005) S. 331-432, hier S. 399 und Abb. 21 - der Beitrag ist auch online verfügbar: URL: <http://www.monasterium.net/ Zajic_Roland.pdf> und in Zukunft DanbuRY, Enluminure (wie Anm. 30) S. 84.

${ }^{35}$ Für England ist vor allem auf die Forschungen und Publikationen von Elizabeth Danbury ; zuletzt Danbury, Décoration (wie Anm. 30). Kurz zusammengefaßt in ZajIC/Roland, Urkundenfälschung (wie Anm. 34) S. 406 f. mit Abb. 21-23, 26. - Die als Abb. 26 abgebildete Urkunde für Merton College (1380 X 5; Oxford, Merton College, MCR 370) wurde dort von der französischen Entwicklung abgeleitet, was sicherlich für den Stil zutrifft. Die ungewöhnliche Anordnung der Figuren - die Universitätsangehörigen knien links außerhalb der Initiale - könnte jedoch Vorbilder bei den illuminierten Avignoneser Bischofssammelindulgenzen haben; ein erstaunlich ähnliche Anordnung findet sich etwa in einem Beispiel vom 22. Jänner 1343 für ein Ölbergrelief an der Magdalenenkapelle bei St. Stephan in Wien (Wiener Diözesanarchiv, sub dato), bei dem die Apostel zu Christus im Binnenfeld aufblicken (siehe monasterium.net und Roland/ZajIC, Chartes médiévales enluminées [wie Anm. 3] Anm. 86 und Abb. 18b).

${ }^{36}$ Dazu ausführlich Brunel, Image du pouvoir (wie Anm. 1) und kurz zusammengefaßt Zajic/Roland, Urkundenfälschung (wie Anm. 34) S. 402-405 mit Abb. 27-32.

${ }^{37} 1367$ I, Paris (ohne Tagesdatum), Paris, Archives nationales, J. 458, Nr. 12: Karl V. und Philipp, Herzog von Orléans, beurkunden Modalitäten der Thronfolge und Bestimmungen die Apanage des Herzogs betreffend; siehe BRUNEL, Image du pouvoir (wie Anm. 1) S. 142-149, Kat.-Nr. 19; Zum Portrait in Büstenform vergleiche auch zwei Urkunden, die 1940 vernichtet wurden; ehem. Tournai, Archives, 1371 II 6, Paris (A. Hocquet, Portraits de Charles V et de Jeanne de Bourbon sur une charte ornée [1371], in: Revue belge d'archéologie et d'histoire de l'art 3 [1933] S. 30-35, mit Abb.) mit unterschiedlichem Rechtsinhalt aber jeweils sehr ähnlicher Gestaltung.

${ }^{38}$ Die Identifizierung mit König Johann dem Guten ist wegen der zweifelhaften Originalität der Aufschrift unsicher. Die physiognomische Ähnlichkeit zwischen dem 
Im 14. Jahrhundert ändert sich die Überlieferungslage grundlegend. Mit den Wappenbriefen (ab 1316) und den illuminierten Bischofssammelindulgenzen (mit figürlichem Schmuck zwischen 1323 und 1363) treten Urkundengattungen auf, von denen sich jeweils mehrere hundert Stücke erhalten haben $^{39}$. Die Kurie wird im 15. Jahrhundert mit den Prunksuppliken und den Kardinalssammelindulgenzen zwei weitere Bestseller kreieren. Als Abb. VIII bilde ich eine Sola Signatura-Supplik für Altenburg, ein heute noch bestehendes Benediktinerkloster in Niederösterreich, $\mathrm{ab}^{40}$. Die Abbildungsvorlage

gut abgesicherten Aussehen Karls V. und dem Dargestellten des Tafelbildes hat u. a. Gerhard Schmidt dazu veranlaßt, auch in dem Tafelbild den Sohn König Johanns des Guten zu sehen: G. ScHMidT, Rezension: Claire Richter Sherman, The Portraits of Charles V of France (1338-1380), New York University Press. New York 1969 (Monographs on Archaeology and the Fine Arts 20), in: Zeitschrift für Kunstgeschichte 34 (1971) S. 72-88, bes. S. 82-87; wiederabgedruckt in: Ders., Malerei der Gotik. Fixpunkte und Ausblicke, hg. von M. Roland, 2 Bde. (2005) hier Bd. 2, S. 311-328, bes. S. 320-326 und 328 (Nachtrag 2003). Der Vater, dessen Gesichtszüge deutlich abweichen, war 1356-60 in englischer Gefangenschaft und kehrte in diese 1364, dem Jahr seines Todes, zurück. Bei dem ungekrönten Dargestellten könnte es sich um Karl als Dauphin handeln. Dann wäre das Bild wohl während der Jahre 1360-64 entstanden, einer Phase der politischen Entspannung. Karl führte zwar nicht die Regierung (vielleicht daher ohne Krone) wie in den Jahren davor und danach, war aber trotzdem politisch und intellektuell führend. Viele Aspekte des Louvre-Portraits (auch jenen einer Spätdatierung, nicht jedoch den hier vorgeschlagenen) behandelt in: S. PERKINSON, The Likeness of the King. A Prehistory of Portraiture in Late Medieval France (2009) zum Bild bes. S. 1-9, 278-303; zur Urkunde von 1367 bes. S. 228-231, 290.

${ }^{39}$ Beide Gruppen werden ausführlich behandelt in Roland/Zajic, Chartes médiévales enluminées (wie Anm. 3); mit zahlreichen Abbildungen.

${ }^{40}$ Die (wie üblich undatierte) Supplik stammt aus dem Pontifikat Papst Eugens IV. (1431-47); vgl. dessen Familienwappen (Condulmaro) und die Tiara in der Initiale. Die Genehmigung der Supplik durch Kardinal Christoph von Rimini schränkt die Zeitspanne auf 1435-44 ein (F. FABIAN, Prunkbittschriften an den Papst [1931] S. 113, Nr. 2). Das durch die Archivsignatur implizierte Datum, nämlich 1300, ist offensichtlich irrig. Die Daten und eine kunsthistorische Beschreibung stehen bei monasterium. net zur Verfügung. - Die durch Tiara und Papstwappen historisierte Deckfarbeninitiale mit Blattgold verfügt über eine Bordüre entlang des linken Randes des Schriftspiegels und der Hälfte des oberen Randes; die vergleichsweise lockeren Fadenranken gehen von Akanthusblättern aus, die sich aus dem Buchstabenkörper entwickeln, und sind mit vor allem kleinen Blättern und gestrichelten Goldscheiben besetzt; als Abschlußmotive auch einfache Blüten. Derartige Fadenranken sind auch in der Buchmalerei durchaus üblich (allgemein verwandt z. B. Rom, Biblioteca Casanatense, Ms. 101, Initiale auf fol. 1r; vgl. den Online-Katalog der Bibliothek mit Abb.). Weitere so frühe Stücke bzw. solche mit unmittelbarer stilistischer Nähe sind mir derzeit unbekannt. Ob sich unter den anderen Prunksuppliken Eugens vergleichbarer Dekor befindet, konnte wegen dem ungenügenden Zugang zu Abbildungen bisher noch nicht geprüft werden. 
stammt aus dem Projekt monasterium.net, das über 250.000 Urkunden kostenfrei online zur Verfügung stellt.

Als Aussteller illuminierter Urkunden begegnen uns Herrscher - vgl. die Angaben zur englischen und französischen Königsurkunde - aber auch „Private“, diese vor allem im deutschsprachigen Gebiet und angrenzenden Regionen Mitteleuropas aber auch in Italien. Daß diese Privaturkunden höchsten Ansprüchen genügen konnten, zeigt exemplarisch eine Urkunde des niederösterreichischen Aufsteigers Caspar von Roggendorf, der „seine“ Pfarrkirche Pöggstall bestiftet (Abb. VII) ${ }^{41}$.

Die behandelten Rechtsgeschäfte reichen von Eheverträgen - neben den beiden bereits erwähnten sei auf eine Ketubba von 1391/92 aus Krems in der Nähe von Wien hingewiesen ${ }^{42}$ - über Dokumente zu Gründungsvorgängen - herausragendes Stück ist der Stiftbrief von Dürnstein ganz in der Nähe von Krems aus dem Jahr $1410^{43}$ - bis zu politisch hochbedeutenden Stücken: Dieser Kategorie gehören der „Zweite geschworene Brief“ der Stadt Zürich von $1373^{44}$, der die Zunftverfassung festschrieb und eine überaus modisch gekleidete Frau mit kühnem Decolleté zeigt, sowie die Veräußerung von Herrschaftsgebieten durch den Bischof von Basel an die Stadt im Jahr $1400 \mathrm{an}^{45}$, entscheidender Rechtsakt für die Entstehung des heutigen Halbkantons Basel-Land. Daß die beiden zuletzt genannten Stücke aus der Schweiz stammen, eine gewisse erotische Komponente zeigen und jeweils der Zusammenhang von Bild und Rechtsinhalt ungeklärt ist, ist erstaunlich aber wohl Zufall.

\section{2) Definition des Untersuchungsgegenstandes}

Im Zusammenhang mit dem hier vorgestellten Forschungsfeld verstehe ich unter Urkunden original vorliegende Rechtsdokumente (Urkundenausfertigungen), die gewissen inhaltlichen und formalen Kriterien gehorchen.

${ }^{41} 1494$ XI 11, Wien; St. Pölten, Diözesanarchiv, Pergamenturkunde sub dato; ausführlich behandelt in Roland/Zajic, Chartes médiévales enluminées (wie Anm. 3) S. 74 .

${ }^{42}$ Das Stück in der Nationalbibliothek in Wien (Cod. Hebr. 218) stellt das einzige illuminierte Beispiel für einen derartigen ganz formelhaften hebräisch abgefaßten Ehevertrag dar; vgl. Roland/Zajic, Chartes médiévales enluminées (wie Anm. 3) S. 66. mit Abb. und der entsprechenden Literatur.

${ }^{43}$ Grundlegend Zajic/Roland, Urkundenfälschung (wie Anm. 34), bes. S. 331-389 (mit Edition und zahlreichen Abbildungen).

${ }^{44}$ Roland/Zajic, Chartes médiévales enluminées (wie Anm. 3) S. 65.

${ }^{45}$ Roland/Zajic, Chartes médiévales enluminées (wie Anm. 3) S. 67 mit Abb. 
Kopialbücher, die ihrerseits oft bedeutenden Buchschmuck aufweisen, sind daher hier nicht in unserem Fokus ${ }^{46}$.

Illuminiert ist ein Begriff der Kunstgeschichte, genauer der Buchmalereiforschung. Er bezeichnet künstlerisch ausgestattete Handschriften und differenziert weder nach Qualität noch nach Umfang der Ausstattung. Neben Prunkhandschriften mit vielfältigem und immer historisiertem Buchschmuck $^{47}$ steht als unteres Ende rein graphischer Dekor ohne inhaltliche Verbindung zum überlieferten Text. Dieser Dekor kann in Bezug auf Umfang und Qualität sehr unterschiedlich sein, von höchster graphischer Finesse bis zu dilettantischem Gekritzel ${ }^{48}$. Daß gerade auch im unfigürlichen Bereich bemerkenswerte Vergleiche möglich sind, belegen die folgenden Abbildungen, deren Fleuronnée augenscheinlich aus derselben, in Köln beheimateten Werkstatt stammt: Die bischöfliche Ablaßurkunde aus dem Jahr 1307

${ }^{46}$ Es gibt einige Fälle, bei denen Kopialbücher auch äußere Merkmale der Originalurkunden mit überliefern. Dies bezieht sich jedoch in der Regel auf graphische Beglaubigungszeichen, am häufigsten Rotae und Monogramme. Einen seltenen Fall, bei dem Original und Kopie überliefert sind, behandelt L. Morelle, De l'original à la copie: remarques sur l'évaluation des transcriptions dans les cartulaires médiévaux, in: Les cartulaires, hg. von O. GuYotjeannin [u. a.] (1993) S. 91-102, bes. Abb. 1-2. Mir ist bisher kein Fall aus dem Mittelalter (sehr wohl aber solche aus dem Barock; vgl. Abb. IXb) bekannt, bei dem Elemente des Dekors illuminierter Originale übernommen wurden.

${ }^{47}$ Dem höchsten Niveau gehört etwa - um ein Beispiel aus meinem unmittelbaren Forschungsschwerpunkt der mitteleuropäischen Buchmalerei der Gotik zu wählen das um 1400 in Wien entstandene Rationale Duranti der österreichischen Herzöge an: Wien, Österreichische Nationalbibliothek, Cod. 2765; grundlegend: Mitteleuropäische Schulen II (ca. 1350-1410). Österreich, Deutschland, Schweiz (Österreichische Akademie der Wissenschaften. Veröffentlichungen der Kommission für Schrift- und Buchwesen des Mittelalters I: Die illuminierten Handschriften und Inkunabeln der Österreichischen Nationalbibliothek 11), bearb. von A. FingernageL, K. Hranitzky, V. Pirker-Aurenhammer, M. Roland und F. Simader (2002) S. 149178 (Kat.-Nr. 31: A. FingernageL) mit Farbabb. 19-26, Abb. 139-167 und Fig. 43-49. Die letzte Ausstattungsstufe des Rationale und der oben erwähnte Dürnsteiner Stiftbrief gehören derselben Stilstufe der Wiener Buchmalerei an.

${ }^{48}$ Dieser graphische Dekor wird - falls er gewissen Prinzipien gehorcht - als Fleuronnée bezeichnet. Dieser Dekorationsstil entwickelt sich im späteren 12. Jahrhundert im nordfranzösischen-flämischen Bereich und wird - mit zahlreichen für die Einordnung wichtigen sowohl lokalen als auch zeitlichen Wandlungen - bis ins beginnende 16. Jahrhundert angewendet. Grundlegend dazu: W. Augustyn, Ch. Jakobi-Mirwald, M. Roland, CH. SAUER, Fleuronné, in: Reallexikon zur Deutschen Kunstgeschichte 9 (Lieferung 105/106, 1996/97) Sp. 1113-1196 (Online verfügbar unter: <http://rdk. zikg.net/gsdl/cgi-bin/library.exe>, Artikelsuche „Fleuronné“); zur Beschreibung

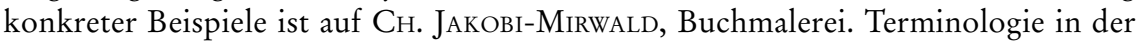
Kunstgeschichte. Dritte, überarbeitete und erweiterte Auflage unter Mitarbeit von M. Roland (2008) S. 65-70 hinzuweisen. 
(Abb. VIa $)^{49}$ dokumentiert eine wichtige Stufe auf dem Weg hin zu den figürlich illuminierten Sammelablässen aus Avignon ${ }^{50}$. Die Vergleichshandschrift, das Wettinger Graduale (Abb. VIb) ${ }^{51}$, wird mit guten Gründen nach 1334 datiert $^{52}$. Wie mit den jeweils sehr zuverlässigen aber weit auseinanderliegenden Datierungen umzugehen ist, bedarf noch weiterer Überlegungen.

491307 XII 28, Köln (Firenze, Archivio di Stato, Diplomatico, S. Pier Maggiore, sub dato). Der Kölner Erzbischof Heinrich II. von Virneburg erteilt den Besuchern jener Kirche, in der Reliquien (zwei Ursulabüsten) Aufstellung finden werden, an genannten Festtagen 40 Tage Ablaß; als Vermittler wird ein Florentiner Bankier genannt (weitere Details in Roland/Zajic, Chartes médiévales enluminées [wie Anm. 3] S. 14f.) - Die Vorlage zu Abb. VIa wurde der digitalen Sammlung des Archivs entnommen (<http:// www.archiviodistato.firenze.it/pergasfi/index.php?opadmin=0\&op=fetch\&type=per gamena\&id=839819>). Das Archivio di Stato di Firenze stellt mehr als 85.000 digitalisierte Urkunden mit über 240.000 Bildern ohne Zugangsbeschränkung im Netz zur Verfügung. Die erweiterte Suche bietet den Begriff „miniatura“ an. Klickt man dieses Feld an, werden 490 Treffer angezeigt (trotz der vorhandenen Digitalisate leider bisher ohne Thumbnails). Alle versuchsweise aufgerufenen Treffer bezogen sich auf Wappendarstellungen, die verso offenbar zu Ordnungszwecken angebracht wurden. Die erwähnte Urkunde mit üppigem Fleuronnée ist - grundsätzlich zu Recht - nicht mit dem Begriff „miniatura“ indiziert. Weiters werden Monogramme, Rotae und andere graphische Beglaubigungszeichen indiziert (nicht jedoch die häufigen Notariatssignete).

${ }^{50}$ Diese Entwicklung ausführlich behandelt und reich illustriert in Roland/ZajIC, Chartes médiévales enluminées (wie Anm. 3) S. 14-21.

${ }^{51}$ Aarau, Aarauer Kantonsbibliothek, MsWettFm 1-3. Die drei heute noch erhaltenen Bände wurden für einen Konvent der Augustinereremiten angelegt; die reiche Deckfarben- und Fleuronnée-Ausstattung stammt von einer Kölner Werkstatt, der auch der 1334 datierte Kasseler Willehalm zuzuordnen ist (Kassel, Landesbibliothek, $2^{\circ}$ Ms. poet. et roman. 1) und gelangten erst spät in das heute namengebende Schweizer Kloster. - Die Vorlage zu Abb. VIb wurde der Datenbank e-codices.ch entnommen (<http://www.e-codices.unifr.ch/de/description/kba/WettFm0001>, einem vorbildhaften Unternehmen, das sich zum Ziel gesetzt hat, das in Schweizer Bibliotheken bewahrte Handschriftenerbe elektronisch verfügbar zu machen. Grundlage der Metadaten bildet (wie vorbildlich angegeben): CH. BRETSCHER-Gisiger/R. GAMPER, Katalog der mittelalterlichen Handschriften des Klosters Wettingen. Katalog der mittelalterlichen Handschriften in Aarau, Laufenburg, Lenzburg, Rheinfelden und Zofingen (2009) S. 156-162.

${ }^{52}$ Das Officium de tempore des Graduales enthält das Dreifaltigkeitsfest am Sonntag nach Pfingsten, das erst 1334 von Papst Johannes XXII. für die Gesamtkirche eingeführt wurde; M. Mollwo, Das Wettinger Graduale. Eine geistliche Bilderfolge vom Meister des Kasseler Willehalmcodex und seinem Nachfolger (1944) bes. S. 5, 74f. und passim. Der Werkstattzusammenhang mit dem Kasseler Willehalm (siehe oben) sichert die Datierung zusätzlich ab. 


\section{Definition 1 \\ Illuminierte Urkunden im weitesten Sinn sind Urkunden mit graphischen oder gemalten Elementen, die nicht der Kontextschrift zuzuordnen sind.}

Damit ist jegliche Form dekorativer Ausgestaltung Teil des Untersuchungsgegenstandes eingeschlossen, auch aufwendige Auszeichnungsschriften und graphische Zeichen und Symbole wie Chrismon, Monogramme oder Notarssignete.

Da eine so weit gefaßte Definition eine nicht handhabbare Fülle an Material generiert, erscheint eine enger gefaßte Definition, die vor allem kunsthistorisch relevante Stücke herausfiltert, sinnvoll:

\section{Definition 2}

Illuminierte Urkunden im engeren Sinn sind Urkunden mit figürlicher (gegenständlicher) Ausstattung, die historisiert ist, also auf Inhalt, Aussteller, Empfänger oder Rezipienten Bezug nimmt. Weiters alle Urkunden, die Farbe(n) prominent in ihr Gestaltungskonzept einbeziehen.

Der erste Teil der Definition schließt rein dekorative figürliche (vor allem auch zoomorphe) Elemente aus, die vielfach zum Standardrepertoire gehören und keine besondere Hervorhebung darstellen. Der zweite Teil der Definition berücksichtigt, daß neben dem Figürlichen zusätzliche Farben das zweite entscheidende Element sind, das illuminierte Urkunden aus der Masse der Überlieferung hervorhebt.

Die Urkunde mit der Gautier, Abt von Chaalis, eine Meßstiftung des französischen Königs Karl V. bestätigt (Abb. XII) ${ }^{53}$, zeigt die außerordentliche Wirkung, die Farben und Goldgrund in der sonst farblosen Welt des Urkundenblattes ausüben obwohl der Dekor unfigürlich ist.

Die Erfahrungen bei der Durchsicht von Beständen (siehe unten) haben uns gelehrt, daß eine so restriktive Definition an den praktischen Anforderungen vorbeizugehen droht. Ich schlage daher eine pragmatische Formulierung vor, die bewußt den für Definitionen üblichen Absolutheitsanspruch ein Stück weit verläßt:

531379 IV 2, Chaalis; Paris, Archives nationales, J 466, n 52: BruneL, Images du pouvoir (wie Anm. 1) Abb. S. 35 und Text S. 50 sowie G. Brunel, Trésor des chartes des rois de France. La lettre et l'image de Saint Louis à Charles VII (2007) S. 93, Kat.Nr. 103. 


\section{Definition 3}

Illuminierte Urkunden sind Urkunden mit graphischen oder gemalten Elementen, die entweder über das allgemein Übliche der Zeit hinausgehen oder einen speziellen Kanzleigebrauch dokumentieren. Besonderes Augenmerk ist auf Urkunden mit figürlicher (gegenständlicher) Ausstattung, die historisiert ist, und auf die Verwendung von Farben zu legen. Weiters sind Urkunden mit graphisch aufwendiger gestalteten Beglaubigungszeichen eingeschlossen.

Gegenüber der weitesten Definition ist die Erfassung von graphischem Dekor nicht absolut definiert, sondern bestimmt sich aus den Gegebenheiten der Entstehungszeit und des Entstehungsorts. Die ist schon deshalb unumgänglich, weil graphisch mitunter exuberant verzierte Urkunden ab dem 15. Jahrhundert vielerorts zum Standard gehören und daher eine vollständige Erfassung dieses Materials als, illuminierte Urkunde' sinnlos erscheint. Andererseits ist es aber zweifelsohne sinnvoll, graphischen (in der Regel gezeichneten, in der Neuzeit vereinzelt auch gedruckten) oder gemalten tintenfarbigem Dekor beispielhaft zu dokumentieren (aber eben nicht flächendekkend zu erfassen); vgl. Abb. IX.

Der folgende Satz der Definition ist - strenggenommen - redundant, da die angesprochenen Phänomene schon vom ersten Teil abgedeckt sind. Er ist aber bedeutsam, weil hier jenes Material benannt wird, das im Zentrum des Interesses steht.

Graphische Beglaubigungszeichen (vor allem Chrismon, Monogramme, Rota, Rekognitionszeichen, Notarssignete) sind für das Medium ,Urkunde typisch und stehen im Schnittpunkt von Rechtssicherung, auf die sie keineswegs reduziert werden dürfen, und angestrebter Wirkung auf den Betrachter, die illuminierte Urkunden grundsätzlich auszeichnet ${ }^{54}$. Der Zusatz „graphisch aufwendiger gestaltet“ ist notwendig, um die Fülle ganz einfacher Zeichen, die die südeuropäische Notariatsurkunde prägen und deren flächendekkende Erfassung in unserem Zusammenhang unrealistisch ist, ausklammern zu können.

\section{3) Wie finde ich illuminierte Urkunden?}

Nachdem wir unseren Gegenstand zuerst an Beispielen kennengelernt und dann definiert haben, können wir uns der Praxis zuwenden. Die Tatsache, daß es bisher keine Versuche einer umfassenden Darstellung der Materie gibt,

${ }^{54}$ Ein kanzleitypisches Beispiel ist Anm. 69 erwähnt, kunsthistorisch interessante Beispiele zeigen Abb. $\mathbf{X}$ und Abb. XIII. 
weist auf ein Kernproblem hin, nämlich die Frage, wie man illuminierte Urkunden überhaupt findet. Außer der in Anm. 1 genannten älteren Literatur und zahlreichen Einzelstudien gibt es kaum zusammenfassende Literatur. Einen systematischen Zugang verfolgten Dénes Radocay, der die ungarischen Wappenbriefe katalogmäßig aufarbeitete ${ }^{55}$, und Homburger und von Steiger, die an Hand eines Avignoneser Ablaßbriefes, diese Großgruppe vorstellten ${ }^{56}$. Ebenfalls an Hand einer Einzelstudie zu dem bereits erwähnten Dürnsteiner Stiftbrief haben Andreas Zajic und ich 2005 versucht einen ersten Überblick zu geben, der bis vor kurzem den umfassendsten Einstieg in die Materie darstellte ${ }^{57}$.

Eine logische Vorgangsweise zur Vervollständigung der Materialbasis wäre es, Urkundenbücher und Archivbehelfe auf illuminierte Beispiele durchzusehen $^{58}$. Die Ergebnisse sind freilich dürftig, da der Schmuck entweder gar nicht erwähnt wird, oder wenn doch, dann in einer erstaunlich unsystematischen Form, jedenfalls wird er nicht registermäßig erfaßt.

$\mathrm{Daß}$ die Digitalisierung von umfangreichen Urkundenbeständen ganz neue Möglichkeiten bietet, ist offensichtlich. Tatsache ist freilich, daß die Metadaten, die online verfügbare Sammlungen erschließen, in fast allen Fällen auf älteren Vorarbeiten aufbauen und daher für unsere Fragestellung kaum wesentlich hilfreicher sind als der Zugang über Urkundenbücher und Archivbehelfe.

${ }^{55}$ D. Radocsay, Gotische Wappenbilder auf ungarischen Adelsbriefen, in: Acta historiae artium Academiae Scientiarum Hungaricae 5 (1958) S. 317-358 (zu den Jahren 1405-1489); DERS., Renaissance letters patent granting armorial bearings in Hungary, in: Acta Historiae Artium Academiae Scientiarum Hungaricae 11 (1965) S. 241-264 (Text) und 12 (1966) S. 71-92 (Katalog zu 1490-1540).

${ }^{56}$ O. Homburger, Ch. von Steiger, Zwei illuminierte Ablassbriefe in Bern, in: Zeitschrift für schweizerische Archäologie und Kunstgeschichte 17 (1957) S. 134-158 mit Taf. 39-50.

${ }^{57}$ Zajic/Roland, Urkundenfälschung (wie Anm. 34). - Aufgrund dieses Artikels wurden wir zu einer Journée d'études (Moyen Âge en images. Les chartes ornées dans l'Europe romane et gothique) nach Paris eingeladen (2007), veranstaltet anläßlich einer Ausstellung an den Archives nationales, die die illuminierten französischen Königsurkunden opulent behandelte: vgl. den bereits 2005 erschienenen Band: Brunel, Images du pouvoir (wie Anm. 1) und das Begleitheft zur Ausstellung: Brunel, Trésor des chartes (wie Anm. 53). Die Vorträge erschienen 2013 in einem Sonderband der Bibliothèque de l'École des chartes. Eine ungekürzte Fassung erschien zeitgleich unter dem Titel „Illuminierte Urkunden des Mittelalters in Mitteleuropa“ im AfD 59 (2013) S. 241-432.

${ }_{58}$ Auch hier ist das Internet und die Digitalisierung von großer Hilfe: vgl. G. VoGELER, Digitale Urkundenbücher. Eine Bestandsaufnahme, in: AfD 56 (2010) S. 363-392. 
Wenn ich etwa in monasterium.net den Suchbegriff „Initiale“ eingebe, erhalte ich 1475 Treffer (davon 911 mit Digitalisaten), also etwas unter 0,59\% $(0,36 \%)$ des Gesamtbestandes von 251.367 digital verfügbaren Urkunden (Stand 20. Jänner 2012). Wären die Ergebnisse in der angezeigten Liste nicht auf ihre Textbotschaft reduziert, sondern von einem Thumbnail des Digitalisats begleitet, könnte man die Treffer effizient durchsehen ${ }^{59}$.

Das Problem ist jedoch nicht die Form der Anzeige, sondern daß nur wenige Treffer den von uns genannten Kriterien entsprechen. Angezeigt werden Freiflächen, in denen die Initialen fehlen, einfache bloß vergrößerte Anfangsbuchstaben, Initialen mit graphischem Dekor und mitunter auch Dinge, die zum Kern unseres Interesses gehören. Auch das wäre irgendwie zu bewältigen, aber die Tatsache, daß Dinge, die zweifelsfrei als illuminiert einzustufen $\operatorname{sind}^{60}$, eben nicht erfaßt werden, entwertet die Ergebnisse. Von zwei Bischofsammelindulgenzen aus dem Jahr 1337 ist bei einer die Suche nach dem Begriff „Initiale“ erfolgreich ${ }^{61}$, die andere fehlt in den Ergebnissen ${ }^{62}$.

${ }^{59}$ Entsprechende Adaptierungen sind bereits angedacht und werden die ersten Früchte einer sehr interessanten Zusammenarbeit mit den Kollegen von monasterium. net sein. Vergleiche auch Abb. XIV für ein Beispiel gelungener Präsentation.

${ }^{60}$ Ich nenne hier ein Notariatsinstrument über einen Kardinalssammelablaß für das niederösterreichische Prämonstratenserstift Geras von 1514 X 16 (Geras, Stiftsarchiv). Wie das Digitalisat zeigt, ist das Stück mit abwechselnd roten und blauen CapitalisBuchstaben in der ersten Zeile, einer rot/blau gespaltenen historisierten Initiale mit Halbfiguren der Apostelfürsten Petrus und Paulus, einem Wappen und einer Darstellung der Sudariums mit Vera Ikon, diese drei in Deckfarbenmalerei, ausgestattet.

${ }^{61} 1337$ VIII 3, Avignon, Bischofssammelindulgenz für die Margarethenkapelle in Göttweig; Göttweig Stiftsarchiv, sub dato: Die Metadaten in monasterium.net stammen aus: A. Fuchs, Urkunden und Regesten zur Geschichte des Benedictinerstiftes Göttweig 1: 1058-1400 (Fontes rerum Austriacarum II/51, 1901) S. 373f. (Nr. 397). Fuchs erwähnt die Initiale folgendermaßen: „Die Urkunde beginnt mit der Initiale U, in welcher ein Miniaturbild ausgeführt ist. Dieses stellt die heil. Maria mit dem Jesukinde dar, vor welcher ein Mönch mit erhobenen Händen betend kniet. Hinter ihr ist eine andere Frauengestalt stehend dargestellt." Wiedergegeben ist offenbar die hl. Margaretha, für deren Altar der Ablaß ausgestellt wurde.

${ }^{62} 1337$ V 15, Avignon, Bischofssammelindulgenz für Pauluskapelle im Wiener Schottenstift; Wien, Schottenstift, Archiv, sub dato (alt Scr. 66, Nr. 13). Die Metadaten in monsaterium.net stammen aus E. HAUswirTH, Urkunden der Benediktiner-Abtei Unserer Lieben Frau zu den Schotten in Wien vom Jahre 1158 bis 1418 (Fontes rerum Austriacarum II/18, 1859) S. 212 f. (Nr. 186). Hauswirth bietet zwar den vollen Text und erwähnt die 14 Siegelreste, die farbig gemalte Initiale mit dem stehenden Christus und den beiden knienden Mönchen ist ihm jedoch nicht bemerkenswert. 


\section{4) Ein Testlauf}

Es führt also kein Weg daran vorbei, die digitalisierten Bilder selbst und nicht nur die Metadaten durchzusehen. Und genau das habe ich mit Hilfe von monasterium.net probiert. Dabei wurde bald klar, daß der Anteil jener Stücke, die kunsthistorisch vor allem interessant sind, verschwindend klein ist ${ }^{63}$. Selbst in einem Bestand wie dem Stiftsarchiv Herzogenburg - insgesamt etwa 2300 Urkunden - fanden sich außer den vier mit dem Dürnsteiner Stiftbrief verbundenen, bereits bekannten Stücken nur drei weitere Beispiele der Kerngruppe: eine Bischofssammelindulgenz von 1341 und zwei neuzeitliche Wappenbriefe. Von den sieben Treffern waren vier bereits bekannt, von den drei Neufunden stammt nur einer aus dem Mittelalter.

Mir schien der Aufwand, den die Probedurchsicht mit sich brachte, für einen so geringen Ertrag zu groß. Andererseits begegneten mir viele graphisch interessante Stücke, die entweder für die jeweilige Kanzlei oder für die Zeit aussagekräftige Dokumente darstellen. Ich entschloß mich daher, nicht nur Material zu erfassen, das der engeren Definition entspricht (so war der ursprüngliche Plan), sondern auch die erwähnten graphisch reicheren (aber weder figürlichen noch farbigen) Stücke und solche mit Beglaubigungszeichen mit einzubeziehen.

$\mathrm{Zu}$ beiden Gruppen möchte ich nun Beispiele vorstellen, um deren Relevanz für unsere Fragestellung zu verdeutlichen:

Für die erste Gruppe steht eine Urkunde Kaiser Friedrichs II. für Göttweig aus dem Jahre 1237, mit der er das niederösterreichische Benediktinerstift unter seinen Schutz stellte (Abb. IXa) ${ }^{64}$. Die FR-Ligatur und andere Lombarden sind mit Palmettenfleuronnée versehen, und stellen damit einerseits eine für das 13. Jahrhundert vergleichsweise reiche graphische Ausstattung von Urkunden dar ${ }^{65}$ und sie sind andererseits typisch für den Kanzlei-

${ }^{63}$ Durchgesehen wurden Bestände aus Niederösterreich bzw. Wien: Aggsbach, Altenburg, Ardagger, Baden, Dürnstein (Chorherren und Klarissin), Geras, Göttweig, Heiligenkreuz, Herzogenburg, Lilienfeld, Seitenstetten, St. Pölten (Chorherren bzw. Diözesanarchiv), Traismauer, Waidhofen an der Ybbs, Wien, Deutschordensarchiv, Wien, Schottenstift, Wien, Wiener Stadt- und Landesarchiv, Wiener Neustadt, Neukloster, und Ybbsitz.

${ }^{64} 1237$ I, Wien (ohne Tagesangabe), Göttweig, Stiftsarchiv, Urk. 118; vgl. monasterium.net.

${ }^{65}$ Ein vertiefender Untersuchungsansatz wäre, ob die Tendenz Urkunden graphisch reicher auszustatten nicht auf päpstliche Urkunden mit durchaus vergleichbarem Dekor reagiert. Erfahrungen mit anderen illuminierten Urkunden haben nämlich gezeigt, daß sehr häufig politische Konflikte zu besonderen Gestaltungen und zu Übernahmen von der Gegenpartei geführt haben. Zu nennen ist hier vor allem die Urkundenpraxis der Kurie und Kaiser Ludwigs des Bayern. 
gebrauch $^{66}$. Sucht man nämlich in monasterium.net nach weiteren Urkunden Friedrichs II. wird man feststellen, daß derartiges Fleuronnée vor allem bei Privilegienbestätigungen für österreichische und bayerische Stifte aus dem Jahr 1237 immer wieder anzutreffen ist (Abb. IXa, c-e) ${ }^{67}$. Neben der Göttweiger Urkunde steht die barocke Kopie (Abb. IXb), die der Göttweiger Archivar Hartmann Dückelmann für seine Materialsammlung angefertigt hat ${ }^{68}$.

Für die zweite Gruppe, die graphischen Beglaubigungszeichen, nenne ich einige Beispiele, die die Breite der Möglichkeiten illustrieren sollen: Zuerst

${ }^{66}$ Zum Kanzleigebrauch bei Friedrich II. vgl. S. GleIXner, Sprachrohr kaiserlichen Willens. Die Kanzlei Kaiser Friedrichs II. (1226-1236) (AfD Beiheft 11, 2006). Gleixner bezieht den graphischen Schmuck als integralen Bestandteil in seine Arbeit ein, der Terminologie und manchen Beurteilungen merkt man freilich an, daß der Autor mit den kunsthistorischen Aspekten der Materie nicht so vertraut ist wie mit den paläographischen. Die hier vorgestellte Gruppe von Fleuronnée-Initialen (ein Terminus den Gleixner nicht verwendet und der die hier auftretenden Formen im Buchwesen bezeichnet) bildet offenkundig einen Neuansatz, denn - trotz der vergleichsweise hohen Konstanz der "Schreiber des graphischen Schmucks“ (so Gleixner) treten die in Abb. IX auftretenden Formen in Gleixners Untersuchung, die bis 1236 reicht, nicht auf. $\mathrm{Ob}$ vielleicht üppigere Fleuronnée-Formen, die Gleixner dem Kontextschreiber Cc zuordnet (S. 276f., vor allem Abb. 511f.) vielleicht schon von dem Florator von 1237 stammen, ist aufgrund der Abbildungsqualität bei Gleixner derzeit nicht zu entscheiden.

${ }^{67}$ Neben dem Göttweiger Diplom (Abb. IXa) und seiner barocken Kopie (Abb. IXb - siehe folgende Anm.) eine Privilegienbestätigung für das Benediktinerinnenstift Erla in Niederösterreich (Abb. IXc - Wien, Haus-, Hof- und Staatsarchiv, AUR, sub dato [1237 I, Wien]), eine Unterschutzstellung für das Benediktinerstift Lambach in Oberösterreich (Abb. IXd - Stiftsarchiv, sub dato [1237 I, Wien]) und Mautfreiheiten für die Zisterze Raitenhaslach in Bayern (Abb. IXe - 1237 III, Wien, München, Bayerisches Hauptstaatsarchiv, KU Reitenhaslach KS 48a). Vergleichbaren Dekor findet sich auf einigen weiteren in Wien ausgestellten Urkunden des Kaisers aus den ersten vier Monaten des Jahres 1237 (Heiligenkreuz, St. Florian, Seitenstetten, Wien, Deutschordensarchiv, Wien, Schottenstift), ist aber offenkundig nicht auf den Ausstellungsort beschränkt. Zur Verwendung von „bildhaften“ Zierelementen und einem Streben nach Ästhetik vgl. W. Koch, Das staufische Diplom. Prolegomena zu einer Geschichte des Urkundenwesens Kaiser Friedrichs II., in: Civiltà del Mezzogiorno d'Italia. Libro, scrittura, documento in età normanno-sveva, hg. von F. D'ORIA (1994) S. 383-424, bes. S. 411/416. Hier wird auch der in Anm. 65 vermutete Zusammenhang mit der päpstlichen Kanzlei thematisiert.

${ }^{68}$ Göttweig, Stiftsbibliothek, Cod. 895 (rot), fol. 30r. Zu Dückelmann (1739-84) und seiner bebilderten Quellensammlung (Cod. 895, 896) vgl. A. ZAJiC, Die Inschriften des Politischen Bezirks Krems (Die Deutschen Inschriften 72 = Die Inschriften des Bundeslandes Niederösterreich 3, 2008) S. L-LI. 
eine ganz kanzleigemäße Schenkung König Heinrichs II. an Stift St. Florian aus dem Jahr 1002 mit Chrismon und Monogramm ${ }^{69}$.

Ein Vertreter der umfangmäßig größten Gruppe, der Notarssignete ${ }^{70}$, ist jenes des in Wien tätigen Nicolaus olim Conradi de Ysenaco (Abb. Xa-d). Die Datierung innerhalb des Signets ist aus diplomatischer Sicht ziemlich ungewöhnlich, uns interessiert aber in erster Linie das (vor allem nördlich der Alpen anzutreffende) gegenständliche Beglaubigungszeichen, hier - wie besonders weitverbreitet - in Monstranzform ${ }^{71}$. Südlich der Alpen ist die Situation grundlegend verschieden: Einerseits ist die Überlieferung dort unvergleichlich umfangreicher, da ein Großteil des Urkundenmaterials von Notaren ausgestellt wurde, andererseits sind die Zeichen in der Regel sehr einfach. Bloß vereinzelte (jedoch sehr spannende) Ausnahmen bestätigen diese Regel: Das Archivio Metropolitano di Bari bewahrt z. B. eine Urkunde auf, auf der das Zeichen des um 1200 in Bari tätigen Protonotars Lupo sichtbar ist, nämlich einen durchaus naturnah gezeichneten Wolf ${ }^{72}$. Die Neapolitaner Notare Dionisio de Sarno und Ruggeriero Pappansogna statteten in den 1420er Jahren Dokumente sogar mit Farbe und figürlichen, mitunter sogar

691002 VII 20, Kirchberg; St. Florian, Stiftsarchiv, sub dato; vgl. MGH, D.H.II.7 und <http://www.hgw-online.net/abbildungsverzeichnis/dh-ii-7>; im von Irmgard Fees betreuten Abbildungsverzeichnis (<http://www.hgw-online.net/abbildungsverzeichnis $>$ ) sind alle Abbildungen zu europäischen Kaiser- und Königsurkunden aufgenommen. Die Urkunde ist in monasterium.net digital verfügbar (mit irrigem Datum: 1002 VII 18). - Daß die beiden hier vorgestellten Gruppen sich überschneiden können, zeigen einige besonders feierliche Urkunden Kaiser Friedrichs II. aus dem Jahr 1237, die von einem Chrismon mit Fleuronnée-Dekor der oben beschriebenen Hand eingeleitet werden.

${ }^{70}$ In diesem Zusammenhang ist auf großangelegte Studien zu Notarszeichen hinzuweisen, die durch derartige Online-Sammlungen kongenial ergänzt werden könnten und die Basis für den bisher fehlenden Gesamtüberblick bilden könnten: diverse Beiträge in Graphische Symbole in mittelalterlichen Urkunden. Beiträge zur diplomatischen Semiotik, hg. von P. RÜCK (Historische Hilfswissenschaften 3, 1996); P.-J. SCHUler, Südwestdeutsche Notarszeichen. Mit einer Einleitung über die Geschichte des deutschen Notarszeichen (1976); E. Reckenzaun, Zur Kunstgeschichte des Notariatssignets in der Steiermark von 1344 bis zur Mitte des 16. Jahrhunderts, in: Mitteilungen des Steiermärkischen Landesarchivs 52-53 (2004) S. 119-155; E. KERN, Notare und Notariatssignete vom Mittelalter bis zum Jahre 1600 aus den Beständen der staatlichen Archive Bayerns (2008).

${ }^{71} \mathrm{Zu}$ diesem Typus und der Frage der Gegenständlichkeit vergleiche eine kurze Bemerkung von P.-J. Schuler, Genese und Symbolik des nordeuropäischen Notarszeichen, in: Graphische Symbole, hg. von RücK (wie Anm. 70) S. 669-687, bes. 671f.

72 Zur Datenbank siehe Anm. 4. Sechs Urkunden seiner Hand sind hier von 1189 bis 1216 nachweisbar. Eine davon zusätzlich mit gezeichnetem, teilweise zoomorphem Dekor (vgl. Anm. 9). 
historisierten Elementen und Wappen aus; ihre Notarssignete, sind ebenfalls farbig und haben die oben erwähnte Monstranzform ${ }^{73}$.

Zuletzt einen Sonderfall aus dem Königreich Leon: Die vom päpstlichen Kanzleigebrauch abgeleitete Rota (rueda; signum rodado) wird vom Siegeltier

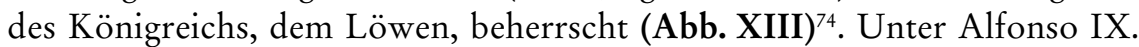
(1188-1230) fallen mitunter die konzentrischen Kreise weg, der Löwe steht dann unvermittelt auf dem Pergament ${ }^{75}$ und ist - obwohl weiterhin „heraldisch" geformt - von einer zoologischen Illustration optisch nicht zu unterscheiden. Das Beglaubigungszeichen nähert sich den Standards der Buchkunst an.

Als Resultat des Testlaufes kann man festhalten: Wenn man die eben umschriebenen Erweiterungen mitberücksichtigt und keine zeitliche Grenze in die Neuzeit festlegt, dann ergeben sich - bezogen auf den Bestand in Herzogenburg - 230 ausgewählte Stücke, ziemlich genau $10 \%$ des digitalisierten Bestandes. Hochgerechnet auf den Gesamtbestand der von monasterium.net digitalisierten Urkunden ergäbe sich eine Sammlung von 25.000 Urkunden.

\section{5) Perspektiven}

a) Ganz schnell und ohne großen Aufwand könnten Thumbnails in die Datenbanken eingebaut werden. Abb. XIV zeigt eine leicht veränderte Ansicht, der Präsentation des Bestandes H 52 (Bemalte Urkunden) des Hauptstaatsarchivs Stuttgart, wo dies bereits vorbildlich gelöst ist ${ }^{76}$.

b) Deutlich aufwendiger ist die Durchsicht der (digitalen) Bestände und das Zusammenstellen der gefundenen illuminierten Stücke zu Gruppen. Eine

${ }^{73}$ In der Datenbank monasterium.net sind sieben Beispiele nachweisbar; eine Fälschung auf das Jahr 1409 X 15, fünf Stücke aus dem Jahr 1423 und eines aus dem Jahr 1426; alle Stücke in der Biblioteca della Società Napoletana di Storia Patria in Neapel (9CCI-27 bis -33). Ich danke Antonella Ambrosio für die Gelegenheit die Stücke im Rahmen der Tagung in Neapel im Original studieren zu dürfen. Vgl. auch Gli inventari dei monasteri di San Pietro a Castello e di San Sebastiano rogati dai notai Dionisio de Sarno e Ruggiero Pappansogna (1423-1426), hg. von S. SEverino (2010).

${ }^{74} \mathrm{Vgl}$. A. Martín Fuertes, El signum regis en el Reino de Léon (1157-1230). Notas sobre su simbolismo, in: Graphische Symbole, hg. von Rück (wie Anm. 70) S. 463478.

${ }^{75}$ Martín Fuertes, Signum regis (wie Anm. 74) S. 473-478.

${ }^{76}$ Das Urkundenselekt wurde 1940 von Max Miller gebildet und in Folge erweitert. Die Online-Präsentation (https://www2.landesarchiv-bw.de/ofs21/olf/struktur.php? bestand=5147\&klassi=\&anzeigeKlassi=001) haben Axel Metz, Martin Schlemmer, Ulrich Schludi, Martin Spiller, Stefan Sudmann und Peter Rückert erarbeitet. 
mögliche Vorgangsweise basierend auf den Beständen von monasterium. net wurde in Abschnitt 4 vorgestellt ${ }^{77}$.

c) Über das Auffinden hinaus muß natürlich auch die Erschließung bedacht werden. Die ausgewählten Stücke müssen mit ganz wenigen Normbegriffen beschlagwortet werden, um so deren graphische bzw. malerische Ausstattung zu erschließen. Ich schlage folgende, einfach durch Ankreuzen auszufüllende Liste vor:

Illuminierte Urkunden im engeren Sinn (Niveau 1)

Historisierter Dekor

$\square$ Miniaturen $\square$ Initialen

$\square$ Randdekor $\square$ Wappen

$\square$ gezeichnet $\square$ gemält

Zusätzliche Farben

Graphisch bemerkenswerte weitere Stücke (Niveau 2)

Gezeichnete Ausstattung (nicht historisiert)

$\square$ Ornamentfelder $\square$ Initialen $\square$ Randdekor

$\square$ Gegenständliche Motive (nicht historisiert)

$\square$ figürlich $\square$ zoomorph $\square$ andere

- Auszeichnungsschrift mit dekorativem Charakter

Graphische Beglaubigungszeichen (Niveau 3)

Chrismon

Monogramm

$\square$ Rota

$\square$ Signum recognitionis

$\square$ Signum notarile

$\square$ Andere Beglaubigungszeichen

$\square$ Anderer Dekor

Urkunden mit diesen Normbegriffen zu versehen, sollte auch kunsthistorischen Laien ohne große Schwierigkeiten möglich sein ${ }^{78}$. Die Supervision

77 Zuletzt haben sich Kontakte zu Kollegen Björn Ommer in Freiburg ergeben, der sich mit Bilderkennung auseinandersetzt. Er geht davon aus, daß man mit beherrschbarem Aufwand diese Auswahl automatisieren könnte, daß also der Computer Urkunden mit Farben, Bildfeldern, graphischem Randschmuck, mit Chrismon, Monogramm, Rota oder Notarssignets erkennen könnte. Ob diese Verlagerung allerdings wünschenswert ist, muß genau überlegt werden, denn eine erneute Durchsicht wäre wohl kaum realistisch. Ein vergleichendes Pilotprojekt sollte aber Teil eines Projektantrages zur Identifizierung illuminierter Urkunden sein.

${ }^{78}$ Nach dem Vortrag in Neapel konnten die Teilnehmer der Tagung einen praktischen Versuch unternehmen. Sie erhielten Abbildungen von vier Urkunden und die obige Liste, wahlweise die in den Sprachen Deutsch, Italienisch, Englisch oder Fran- 
und Beurteilung im Detail sowie das ikonographische Register müssen aber natürlich dem ausgebildeten Kunsthistoriker vorbehalten bleiben, um Einheitlichkeit und qualitativen Standard zu garantieren.

Nach Abschluß der Arbeiten steht eine grob in Gruppen geordnete Bildersammlung zur Verfügung mit graphisch bemerkenswerten Beispielen und Rekognitionszeichen, der in weiteren Schritten wissenschaftlich strukturiert und ausgewertet werden kann.

d) Unabhängig von der Bestandsdurchsicht sollte man gezielt illuminierte Urkunden im engeren Sinn sammeln. Dazu sind bereits erhebliche Vorarbeiten geleistet: Andreas Zajic und ich haben eine elektronische Materialsammlung aufgebaut, die über 400 Beispiele dokumentiert, wobei sich durch die systematische Einarbeitung aller jener Stücke, die in der von uns gesammelten Literatur erwähnt werden, der Bestand wohl zumindest verdoppeln ließe, freilich vor allem durch zusätzliche Bischofssammelindulgenzen und Wappenbriefe. Ich kann mir gut vorstellen, daß in absehbarer Zeit vielleicht 1000 oder 1500 illuminierte Urkunden elektronisch verzeichnet sein werden, die einen Großteil der illuminierten Urkunden im engeren Sinn bis ca. 1520 umfassen.

\section{6) Wem nützt die Kenntnis illuminierter Urkunden?}

Offensichtlich der Kunstgeschichte, denn sie erhält Zugang zu neuem, in der Regel sowohl lokalisiertem als auch datiertem Material. Zu welch spannenden Fragen diese urkundenspezifischen Fakten führen können, habe ich oben an einem Beispiel demonstriert (siehe S. $257 \mathrm{f}$. und Abb. VIa und Abb. VIb).

Die Katalogisierung illuminierter Urkunden nützt den Archiven, denn ihnen steht ein wissenschaftlich aufgearbeiteter Bestand an optisch interessantem Material zur Verfügung, der z.B. für Ausstellungsanfragen genutzt werden kann.

Die Diplomatik hat bisher vergleichsweise wenig Interesse an äußeren Merkmalen der Urkunden gezeigt, zumal wenn diese nicht der Beglaubigung dienen. Dabei sollte sie sich schon längst gefragt haben, warum manche Stücke so prächtig ausgestattet wurden. Dies geschah wohl kaum als l'art pour l'art. Kaiser Ludwig der Bayer dokumentierte ohne ein Wort seine hervorgehobene Stellung durch die Identifikation mit dem siegreichen Adler $(\mathrm{Abb} . \mathrm{XV})^{79}$. Die Darstellung des Kirchenpatrons auf einem illuminierten

zösisch, und konnten ankreuzen; siehe <http://www.cei.lmu.de/digdipl11/slides-rep/ Roland/Bsp.pdf>

${ }^{79}$ Die grundlegende Studie zu den illuminierten Urkunden Kaiser Ludwigs stammt

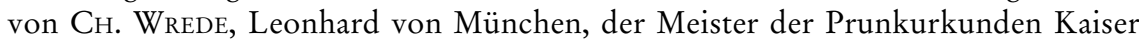


Sammelablaß - offensichtlich für jeden Kirchenbesucher - hat die Freigiebigkeit des bußfertigen Besuchers, durch den Ablaß befreit von befürchteter Strafe, sicherlich erheblich gesteigert und hat so die zusätzlichen Herstellungskosten eines illuminieren Stückes gerechtfertigt. Der soziale Aufsteiger hat seine Kollegen (abends bei einem Glas Wein) mit einem schön gemalten und bunten Wappenbrief beeindruckt, ein neu gegründetes Kloster hat sich mit einem repräsentativen Stück ein Identifikationsobjekt - gleichsam als Ersatz für lange Tradition - zugelegt.

So modern das alles klingt, diese mediale, auf den Betrachter fokussierte Komponente kennt die Diplomatik schon lange: Freilich wurde dies - zumindest bei meinen Diplomatik- bzw. Paläographievorlesungen - an Hand der litterae caelestes ${ }^{80}$ abgehandelt, also einer spätantiken (2.-5. Jh.) kaiserlichen Reservatschrift für Urkunden auf Papyrus.

\section{7) Schlußwort}

Illuminierte Urkunden wirkten auf Zeitgenossen, auf mittelalterliche Nachnutzer und sie wirken - wie der Erfolg bei Ausstellungen zeigt - auch auf heutige Betrachter. Diese Photomontage (Abb. XI) kombiniert einen Ferrari und einen Fiat Cinquecento mit dem noch ganz schmucklosen Wappenbrief des ungarischen Königs Ludwig des Großen für Kaschau/Kosice von 1369 und dessen Bestätigung durch Sigismund von 1423, jetzt eine Pracht, von einem führenden Buchmaleratelier der Zeit ausgeführt ${ }^{81}$. Beide Urkunden besagen weitgehend dasselbe; auch mit beiden Fahrzeugen gelangt man von A nach B, bloß „WIE“, das ist die entscheidende Frage.

Die Chancen, die sich durch die Erforschung illuminierter Urkunden bieten, liegen im kreativen Potential, das sich aus der impliziten Spannung zwischen dem Rechtsdokument Urkunde und dem Bild bzw. Kunstwerk mit dessen medienspezifischer Ausrichtung auf den Betrachter hin ergibt.

Wenn man also die beschriebenen Maßregeln befolgt, besteht die realistische Chance mit überschaubarem und kalkulierbarem Aufwand ein Material aufzubereiten, das in seiner gesamteuropäischen Dimension und durch seinen viele Teildisziplinen beschäftigenden Charakter der Mediävistik neue Impulse geben kann.

Ludwigs des Bayern (1980); diese Urkunde als Kat.-Nr. 17 behandelt.

${ }^{80}$ O. Kresten, Zur Frage der "Litterae caelestes", in: Jahrbuch der österreichischen byzantinischen Gesellschaft 14 (1965) S. 13-20; vgl. auch B. Bischoff, Paläographie des römischen Altertums und des abendländischen Mittelalters (1979) S. 83.

${ }^{81}$ Die beiden städtischen Wappenbriefen und die kunsthistorische Einordnung des Schmucks der Urkunde von 1423 ausführlich behandelt in Roland/ZajIC, Chartes médiévales enluminées (wie Anm. 3). 


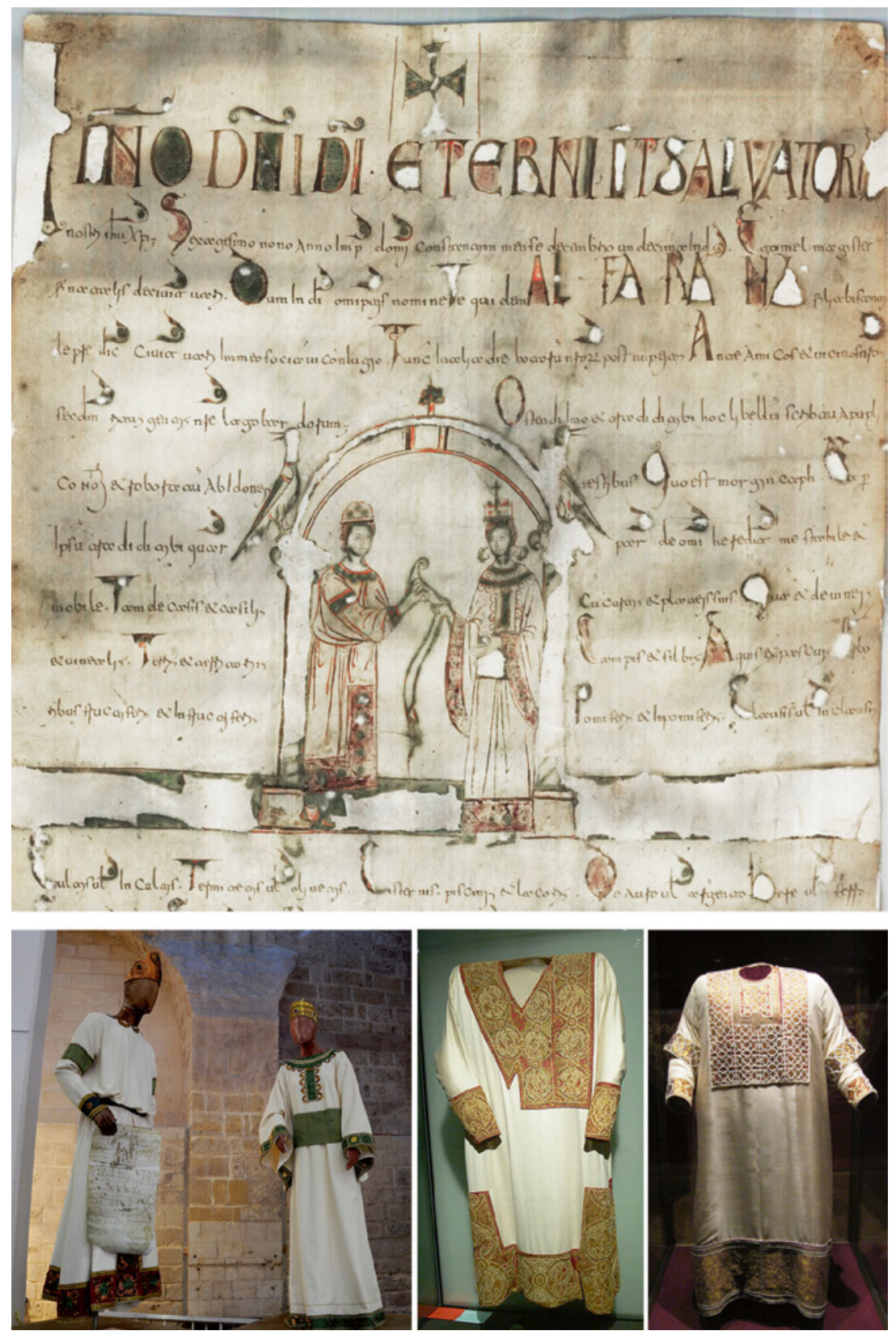

Abb. IVa: Detail der Hochzeitsurkunde des Magister Mel und der Alfarana mit der Darstellung des Brautpaares (1028 XII, Bari - siehe Anm. 4) - Abb. IVb: Rekonstruktion anläßlich einer Ausstellung in Bari 2011 (siehe Anm. 29) - Abb. IVc: Sogenannte Tunika Heinrichs II. (wohl 1. Viertel 11. Jh.; Bamberg, Domschatz) Abb. IVd: Sogenannte Alba der Reichskleinodien (Sizilien, 1181). 


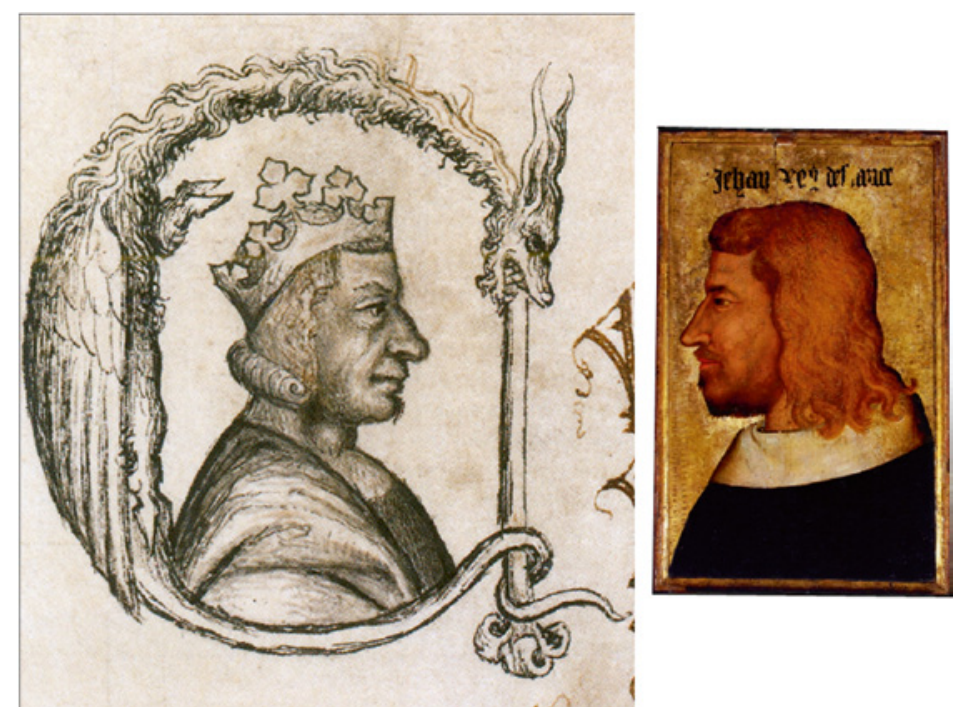

Abb. Va: Detail der Urkunde König Karls V. von Frankreich und Herzog Philipps von Orléans mit der historisierten Dracheninitiale mit Büste des Königs (1367 I, Paris - siehe Anm. 37) - Abb. Vb: Portrait, wohl irrig bezeichnet als Jehan roy de France (Paris, Louvre).

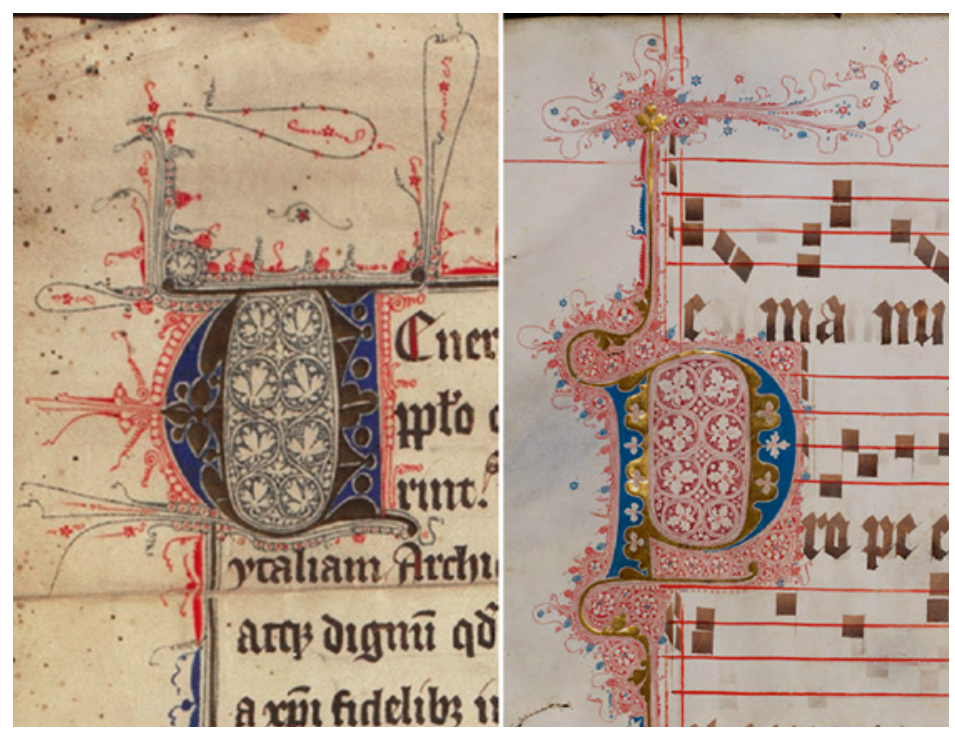

Abb. VIa: Fleuronnée-Initiale eines Ablasses des Kölner Erzbischofs Heinrich II. von Virneburg (1307 XII 28, Köln - siehe Anm. 49) Abb. VIb: Wettinger Graduale, Fleuronnée-Initiale, Köln wohl nach 1334 (Aarau, Aargauer Kantonsbibliothek, MsWettFm1, fol. 4v - siehe Anm. 51). 


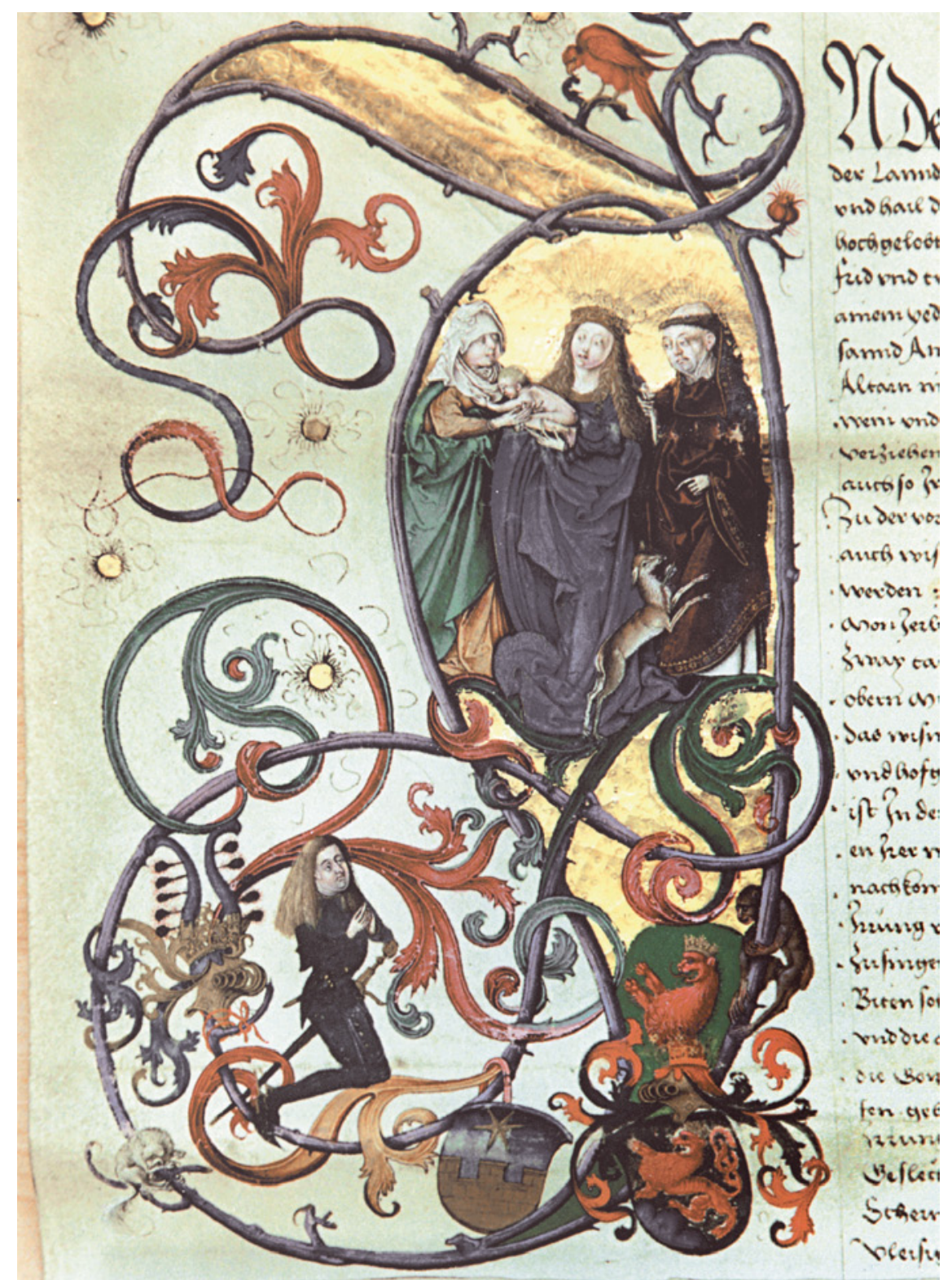

Abb. VII: Detail des Stiftbriefes des Kaspar von Roggendorf für die Pfarrkirche Pöggstall in Niederösterreich (1494 XI 11, Wien - St. Pölten, Diözesanarchiv, Pergamenturkunde sub dato - siehe Anm. 41). 


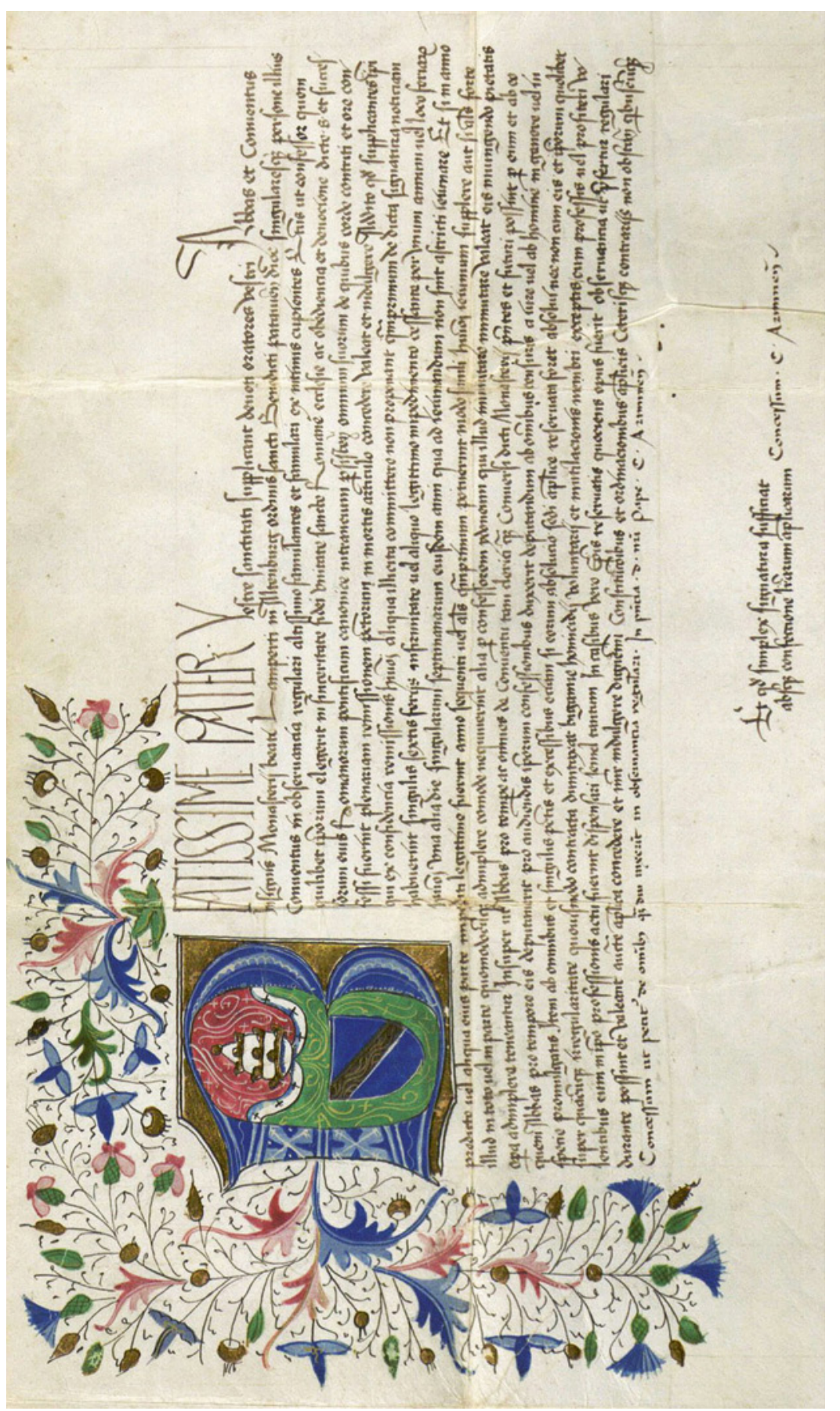

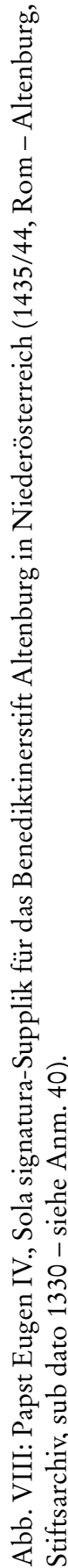



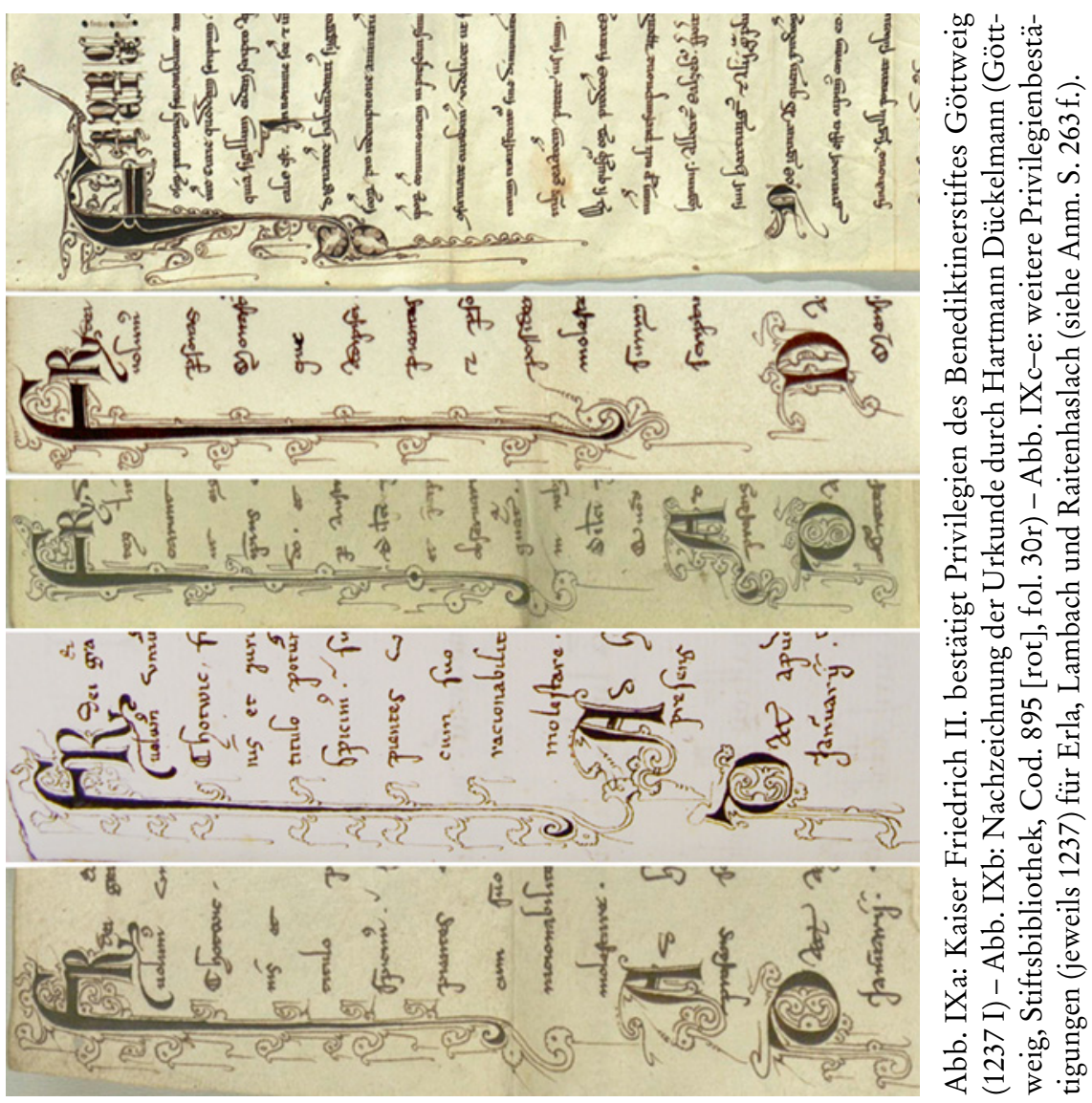
Colour plates: Roland
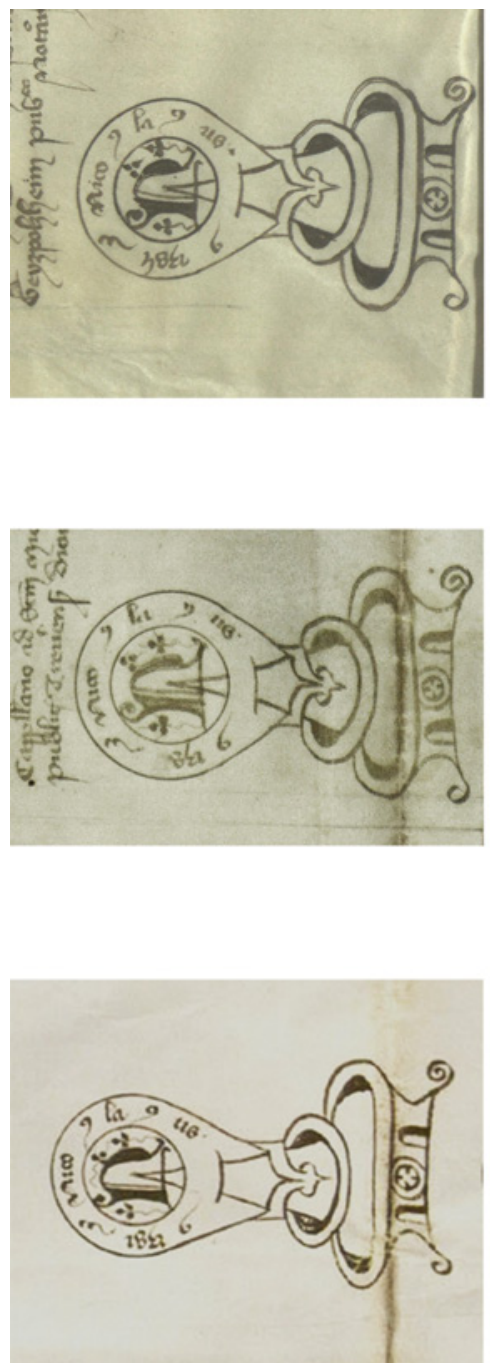
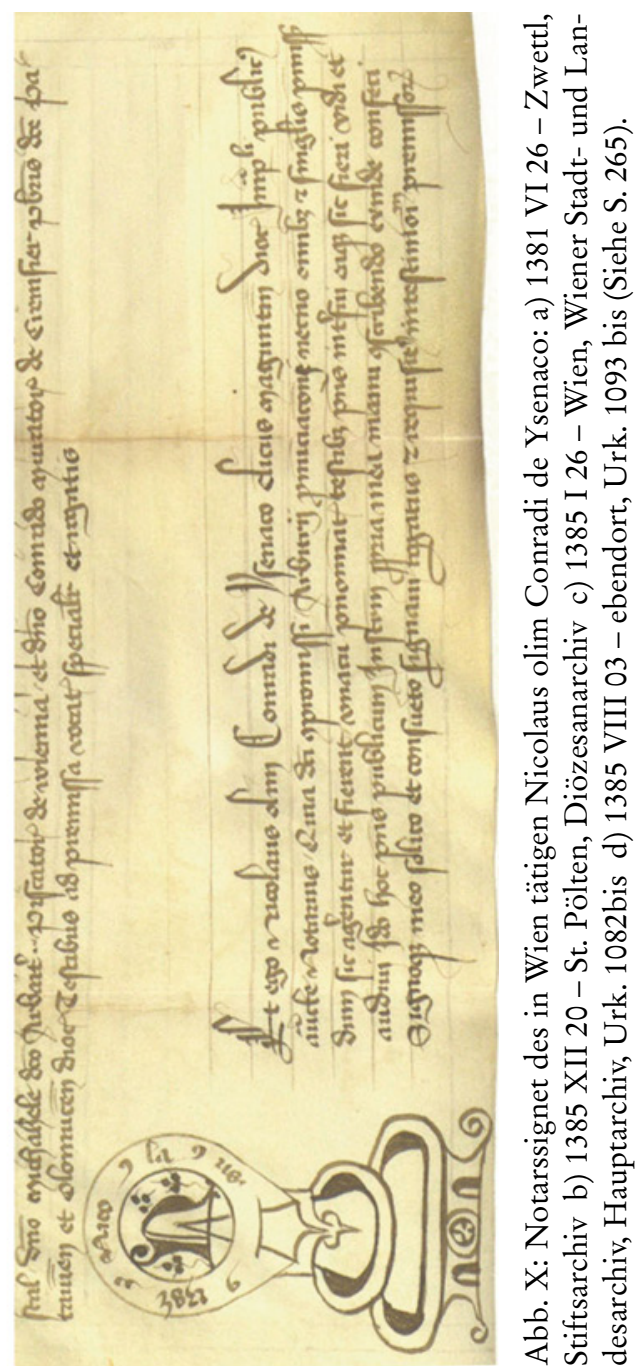


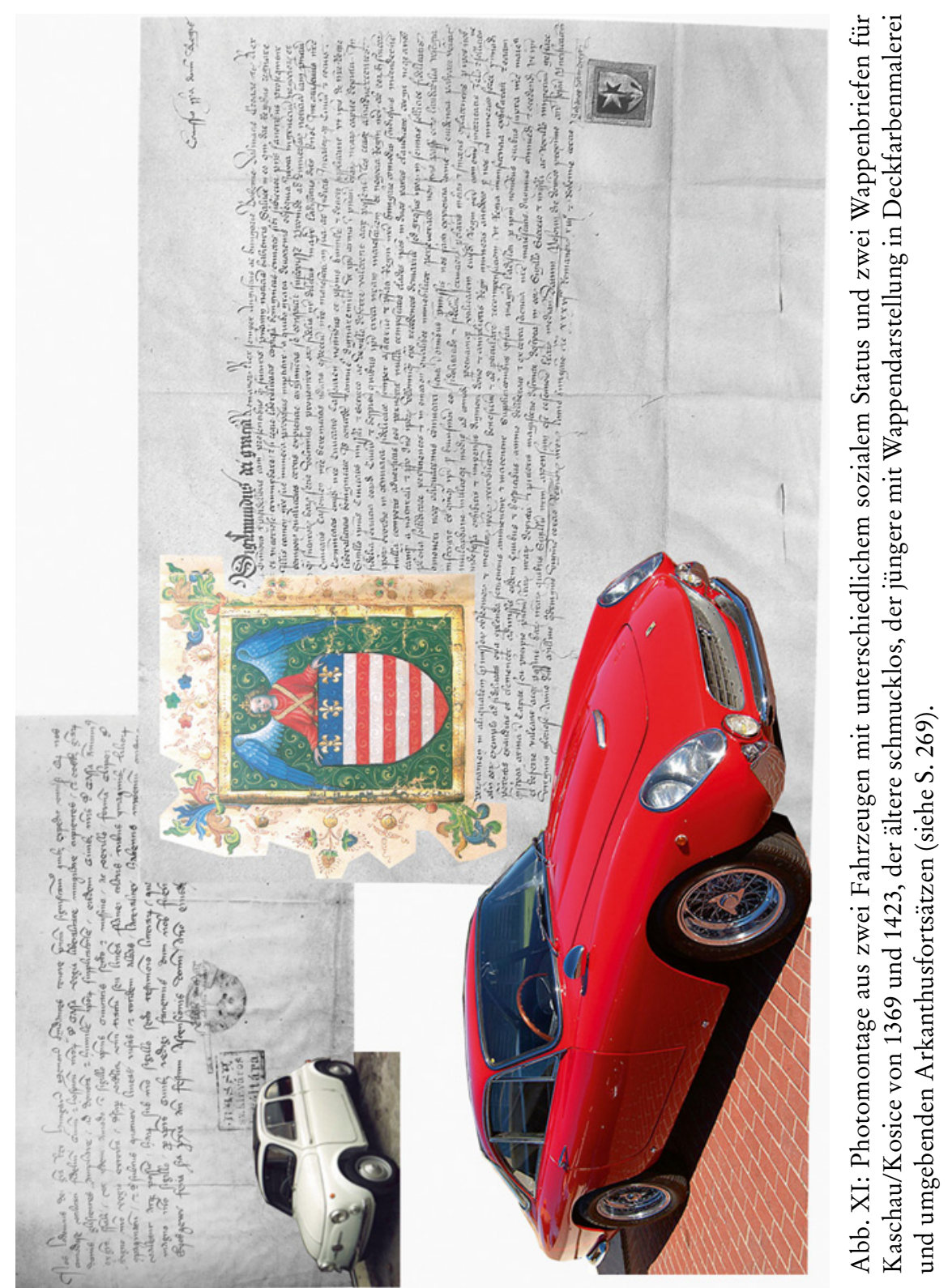




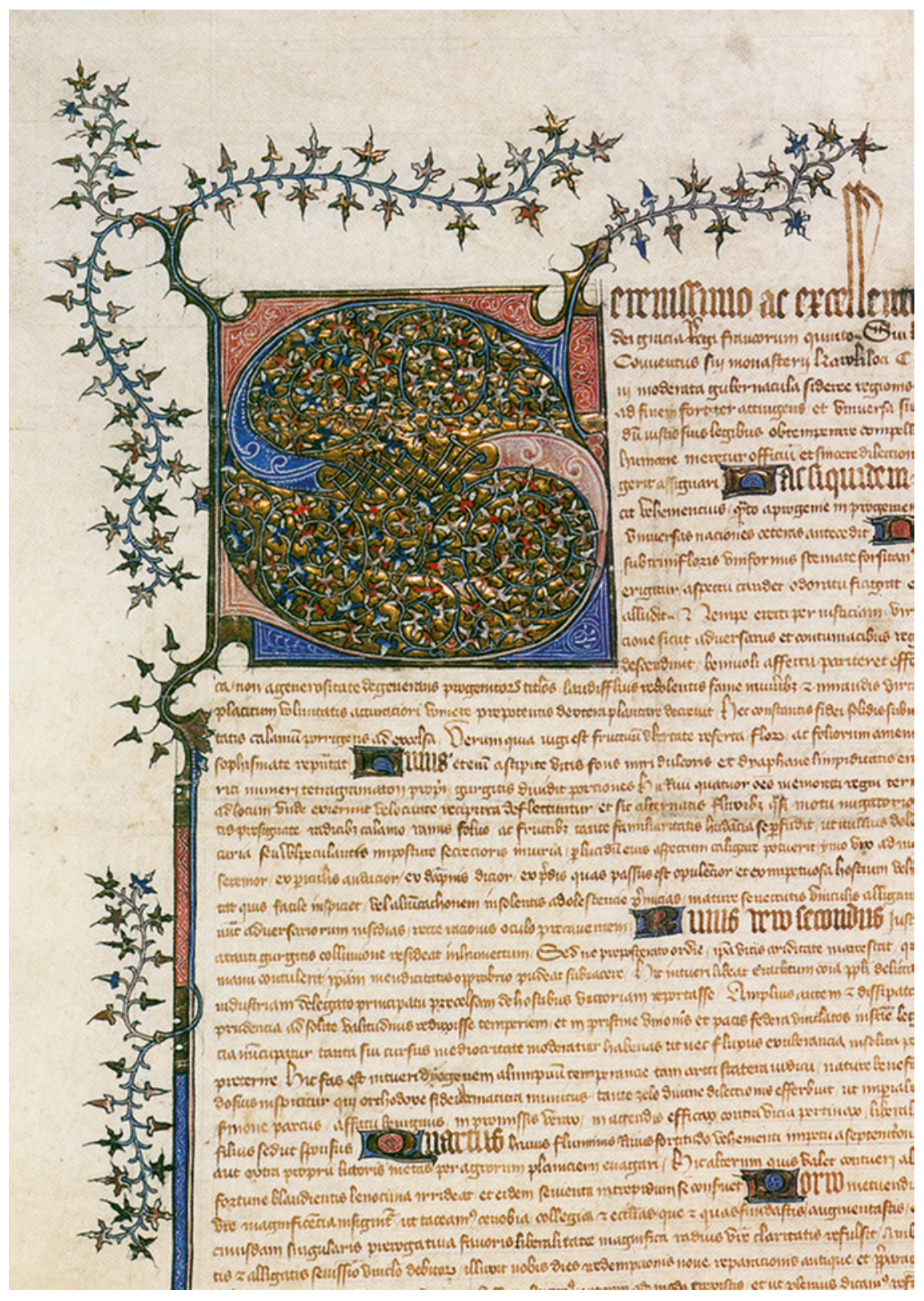

Abb. XII: Der Abt von Chaalis bestätigt eine Meßstiftung Karls V. von Frankreich (1379 IV 2, Chaalis - siehe Anm. 53). 

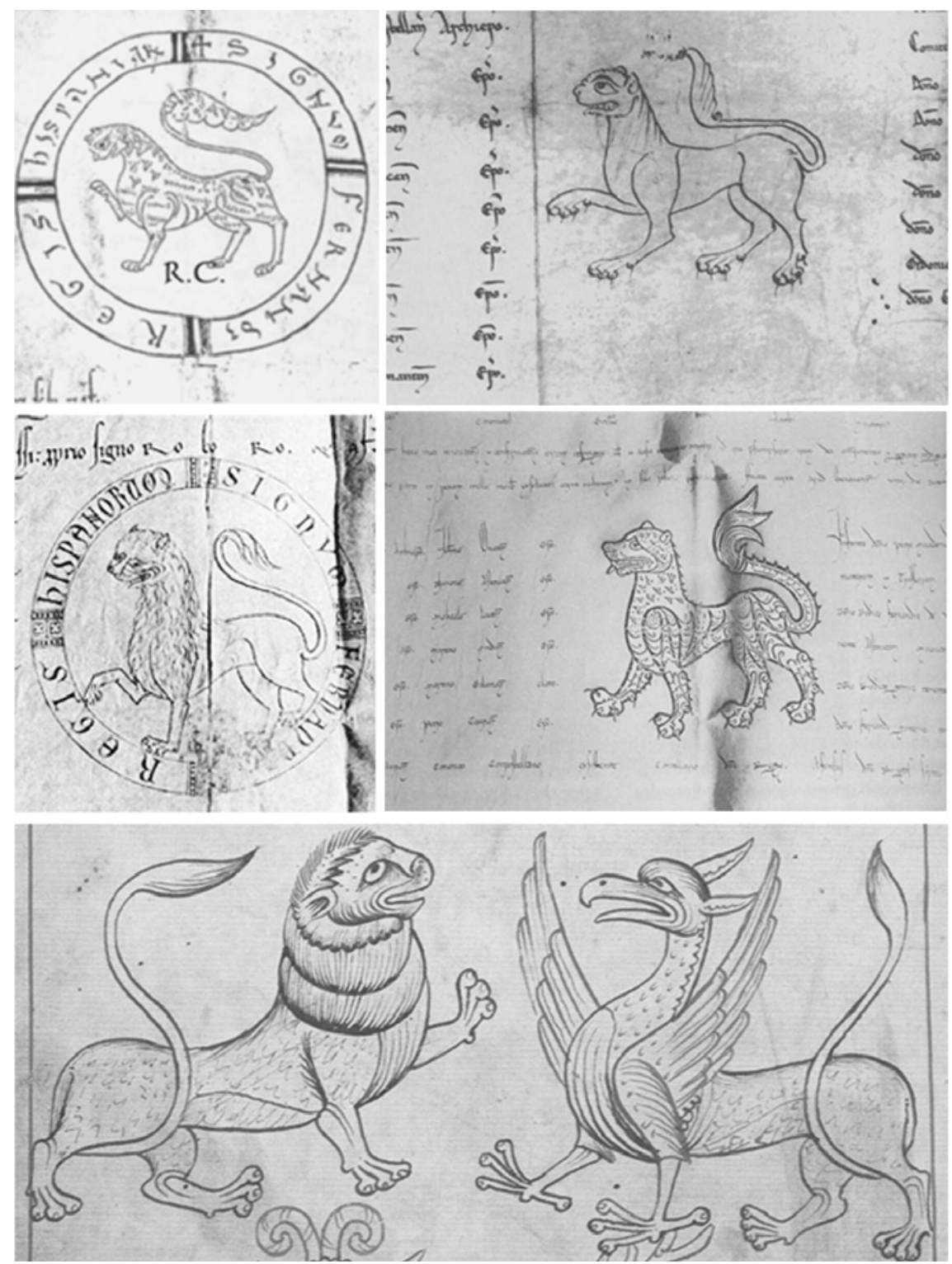

Abb. XIII: Rotae aus vier Königsurkunden aus Leon (zwei mit $[1165,1186]$ und zwei ohne umgebende Kreise [1219, 1229]) und Detail aus dem Musterbuch von Rein (Wien, Österreichische Nationalbibliothek, Cod. 507, fol. 8v), Österreich, wahrscheinlich ein Heiligenkreuzer Zeichner, um 1220/30 (siehe S. 266). 


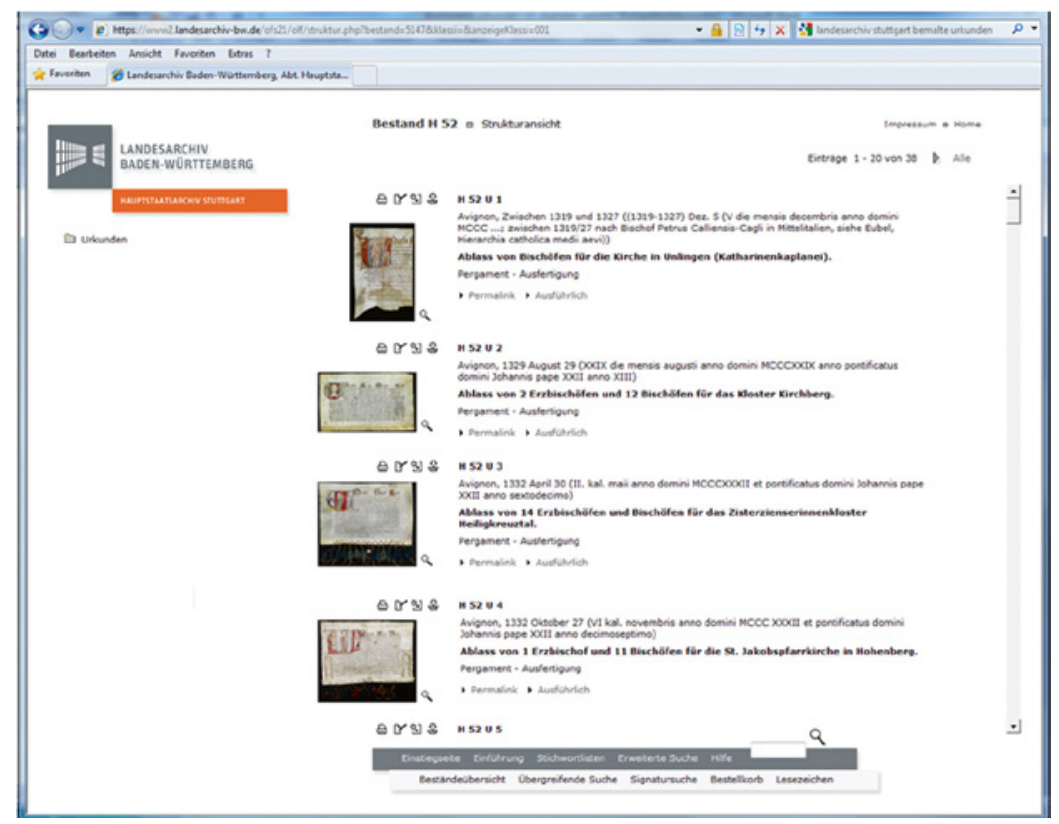

Abb. XIV: Bearbeiteter Screenshot der Urkundengruppe H 52 (bemalte Urkunden) aus dem Hauptstaatsarchiv Stuttgart. Die Kombination von Thumbnail und Kurzbeschreibung ermöglicht die effiziente Durchsicht von Beständen (siehe Anm. 76).

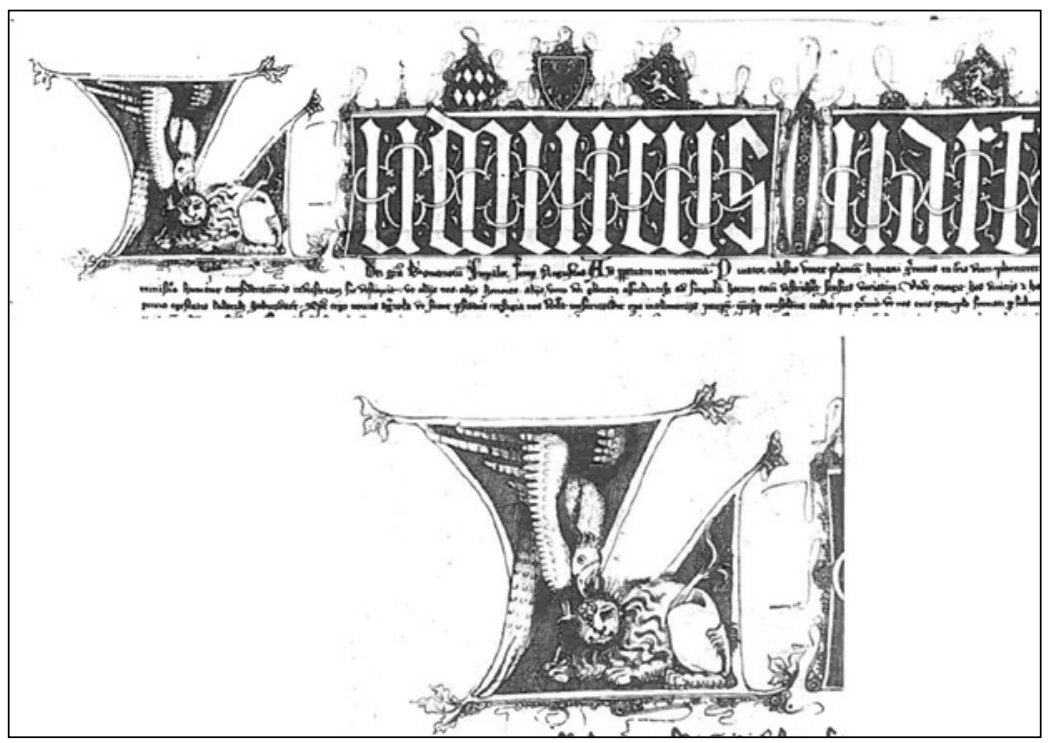

Abb. XV: Kaiser Ludwig der Bayer für das Nürnberger Heilig Geist-Spital (1341 II 24 - Nürnberg, Staatsarchiv, Rep. 1a, Kaiserl. Priv. Nr. 51 - siehe Anm. 79). 